\title{
Adaptive Bandwidth Reservation and Admission Control in QoS-Sensitive Cellular Networks
}

\author{
Sunghyun Choi, Member, IEEE, and Kang G. Shin, Fellow, IEEE
}

\begin{abstract}
How to keep the probability of hand-off drops within a prespecified limit is a very important Quality-of-Service (QoS) issue in cellular networks because mobile users should be able to maintain ongoing sessions even during their hand-off from one cell to another. In order to keep the hand-off dropping probability below a prespecified target value (thus providing a probabilistic QoS guarantee), we design and evaluate predictive and adaptive schemes for bandwidth reservation for the hand-offs of ongoing sessions and the admission control of new connections. We first develop a method to estimate user mobility based on an aggregate history of hand-offs observed in each cell. This method is then used to probabilistically predict mobiles' directions and hand-off times in a cell. For each cell, the bandwidth to be reserved for hand-offs is calculated by estimating the total sum of fractional bandwidths of the expected hand-offs within a mobility-estimation time window. Three different admission-control schemes for new connection requests using this bandwidth reservation are proposed. We also consider variations that utilize the path/location information available from the car navigation system or global positioning system (GPS). Finally, we evaluate the performance of the proposed schemes extensively to show that they meet our design goal and outperform the static reservation scheme under various scenarios.
\end{abstract}

Index Terms-Wireless/mobile cellular networks, predictive and adaptive bandwidth reservation, mobility estimation, admission control, QoS guarantees, connection blocking probability, and hand-off dropping probability.

\section{INTRODUCTION}

$\mathrm{R}$ ECENTLY, there has been a rapid growth of efforts in research and development to provide mobile users the means of "seamless" communications through wireless media. This has made it possible to implement and deploy the current cellular systems, PCS (Personal Communication Systems), and wireless LANs [1]. There has also been a great demand for broadband multimedia communication involving digital audio and video. A number of researchers have been looking into communication services with guaranteed QoS, such as delivery delay and link bandwidth in wired networks [17], [22]. Limited efforts to support QoS guarantees in wireless/mobile. In addition to packet-level QoS issues (related to packet-delay bound, throughput, and packet-error probability) considered in [2], [3], connectionlevel QoS issues (related to connection establishment and management) need to be addressed, as users are expected to move around during communication sessions, causing hand-offs between cells. The current trend in cellular networks is to reduce cell size to accommodate more mobile users in a given area, which will, in turn, cause more frequent hand-offs, thus making connection-level QoS even more important.

- S. Choi is with Wireless Communications and Networking, Philips Research, 345 Scarborough Rd., Briarcliff Manor, NY 10510.

E-mail: sunghyun.choi@philips.com.

- K.G. Shin is with the Real-Time Computing Laboratory, EECS Department, University of Michigan, 1301 Beal Ave., Ann Arbor, MI 481092122. E-mail: kgshin@eecs.umich.edu.

Manuscript received accepted 8 Apr. 2002.

For information on obtaining reprints of this article, please send e-mail to: tpds@computer.org, and reference IEEECS Log Number 116279.
One of the most important connection-level QoS issues is how to control (or reduce) hand-off drops due to lack of available channels in the new cell, since mobile users should be able to continue their ongoing sessions. We will therefore consider two connection-level QoS parameters: the probability $P_{C B}$ of blocking new connection requests and the probability $P_{H D}$ of dropping hand-offs. Ideally, we would like to avoid hand-off drops so that ongoing connections may be preserved as in a QoS-guaranteed wired network. However, this requires the network to reserve bandwidth in all cells a mobile might pass through; this is not possible in most cases, because the mobile's direction is not known a priori. Moreover, this perconnection/mobile reservation will severely underutilize and, hence, quickly deplete, bandwidth, which will, in turn, lead to a high $P_{C B}$.

Each cell can, instead, reserve fractional bandwidths of ongoing connections in its adjacent cells, and this aggregate reserved bandwidth (for multiple ongoing connections) can be used solely for hand-offs, not for new connection requests. The problem is then how much of bandwidth in each cell should be reserved for hand-offs. In this paper, we present a predictive and adaptive scheme for bandwidth reservation and admission control that keeps the hand-off dropping probability below a target value, $P_{H D \text {,target }}$. Since it is practically impossible to completely eliminate hand-off drops, the best one can do is to provide some form of probabilistic QoS guarantees by keeping $P_{H D}$ below a prespecified value. Our scheme is predictive as it estimates the directions and hand-off times of ongoing connections in adjacent cells, and is also adaptive because it dynamically 


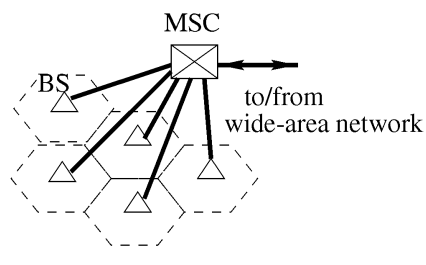

(a)

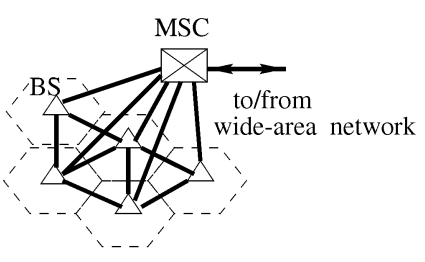

(b)

Fig. 1. Communication architectures among the MSC and BSs: (a) star topology with the MSC at the center and (b) fully-connected network.

adjusts the bandwidth reserved according to the estimation results and the observed hand-off dropping events.

We also address how to utilize the next-cell information on which cell a mobile will move into after departing from its currently residing cell, if and when such information is available to the system. This information can be extracted when the path of the mobile is known, e.g., from an Intelligent Transportation Systems (ITS) navigation system [21], or predicted with high accuracy when the location of the mobile is known, e.g., from the global positioning system (GPS). How to predict the next cell of a mobile from its location information will be addressed in Section 5. As will be shown later, the path/location information-aided schemes can meet the design goal while admitting more new connections into the system even with much less computational complexity than those other schemes without path/location information.

The rest of this paper is organized as follows: Section 2 describes the system specification and states the assumptions to be used. The users' mobility estimation based on an aggregate history of observations is presented in Section 3. Section 4 describes the proposed predictive, adaptive bandwidth reservation and three admission-control schemes with and without next-cell information. Section 5 presents and discusses the comparative evaluation results of the proposed and static-reservation schemes under various scenarios. Section 6 discusses related work, putting our scheme in a comparative perspective. Finally, the paper concludes with Section 7.

\section{System Model}

We consider a wireless/mobile network with a cellular infrastructure, comprising a wired backbone and a (possibly large) number of base stations (BSs) or access points (APs). The geographical area controlled by a BS is called a cell . A mobile, ${ }^{1}$ while staying in a cell, communicates with another party, which may be a node connected to the wired network or another mobile, through the BS in the same cell. When a mobile moves into an adjacent cell in the middle of a communication session, a hand-off will enable the mobile to maintain connectivity to its communication partner, i.e., the mobile will start to communicate through the new BS, hopefully without noticing any difference.

A hand-off could fail due to insufficient bandwidth available in the new cell, and in such a case, a connection

1. We use the term "mobiles" to refer to mobile or portable devices, e.g., hand-held handsets or portable computers. hand-off drop occurs. Here, we preclude 1) delay-insensitive applications, which might tolerate long hand-off delays in case of insufficient bandwidth available in the new cell at the time of hand-off and 2) soft hand-off of the Code Division Multiple Access (CDMA) systems [20], [4], in which a mobile can communicate via two adjacent BSs simultaneously for a while before the actual hand-off takes place. We propose to set aside some bandwidth in each cell for possible hand-offs from its adjacent cells. This reserved bandwidth can be used only for hand-offs from adjacent cells, but not for admitting newly-requested connections in the cell. A connection is specified by its required bandwidth, ${ }^{2}$ and a newly-requested connection in a cell requires a very simple admission test:

$$
\sum_{i} b_{i}+b_{\text {new }} \leq C-B_{r}
$$

where $C$ is the wireless link capacity, $B_{r}$ is the target reservation bandwidth, i.e., the required bandwidth to be reserved for hand-offs, $b_{i}$ is the bandwidth being used by an ongoing connection $i$, and $b_{\text {new }}$ is the bandwidth required by the newly-requested connection. Upon arrival of a new connection request, $B_{r}$ is updated predictively and adaptively-before performing the admission test (1) on the request-depending on the traffic status in adjacent cells. Note that $B_{r}$ is a target, not the actual reserved bandwidth, since a cell may not be able to reserve the target bandwidth, i.e., $\sum_{i} b_{i}+B_{r}>C$. This can happen because a BS can control the admission of only newly-requested connections, not those connections handed off from adjacent cells.

Our bandwidth reservation is based on information from adjacent cells such as the number of ongoing connections and their bandwidth requirements. Thus, it is very important to maintain inter-BS communications. The underlying network topology for BSs can have mainly two possible configurations as shown in Fig. 1. There is a node called "Mobile Switching Center" (MSC), which covers a number of BSs, and works as a gateway to and from the wide area network. Fig. 1a shows a star-topology interconnection among the MSC and BSs, in which there are no direct connections among BSs. This is a typical structure found in the currently-deployed cellular networks. In this environment, each BS delivers the information about existing connections in its cell to the MSC. The MSC

2. A connection in QoS-sensitive networks might be specified by its required buffer space as well as bandwidth. However, in wireless networks, bandwidth (of wireless links) is the most precious resource, so we consider the bandwidth reservation only. Buffer space reservation can be treated similarly to the bandwidth reservation considered here, and admission control can be integrated with this buffer reservation. 


\begin{tabular}{|c|}
$\mathrm{C} 1$ \\
\hline $\mathrm{C} 0$ \\
\hline $\mathrm{C} 2$ \\
\hline
\end{tabular}

(a)

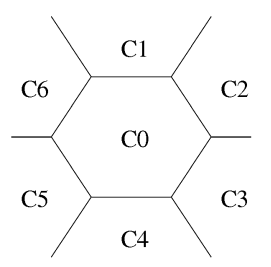

(b)
Fig. 2. Indexing of cells. (a) one-dimensional case. (b) two-dimensional

will then determine the target reservation bandwidth in each cell, and accordingly, will perform the admission test for each newly-requested connection in a cell within its coverage. On the other hand, Fig. $1 \mathrm{~b}$ shows the case where BSs are fullyconnected. In this topology, BSs can communicate directly, not via the MSC, and each BS can determine the target reservation bandwidth and, hence, perform the admission test for each newly-requested connection in its cell.

All cells around each cell $A$ are indexed: ${ }^{3} A$ with 0 , and the others with numbers beginning with 1 as shown in Fig. 2. Let $C_{i, j}$ be connection $j$ in cell $i$ and $b\left(C_{i, j}\right)$ be its required bandwidth. For simplicity, we assume that a mobile cannot have multiple connections simultaneously, so by an active mobile, we mean a mobile with one existing connection. ${ }^{4}$ The cellular system uses a fixed channel allocation (FCA) scheme, and cell $i$ has a wireless link capacity $C(i)$. The unit of bandwidth is $\mathrm{BU}$, which is the required bandwidth to support a voice connection. A connection runs through multiple wired and wireless links and, hence, we need to consider bandwidth reservation on both wireless and wired links for hand-offs. However, we will confine ourselves to reservation of wireless link bandwidth in each cell, because routing and/or rerouting upon hand-off of a connection is beyond the scope of this paper. Our scheme can be extended easily to include wired link bandwidth reservation by considering the routing and rerouting inside the wired network.

\section{Mobility Estimation}

We probabilistically model mobiles' hand-off behaviors and estimate their mobility based on an aggregate history of hand-offs observed in each cell. In order to understand the rationale behind our mobility estimation, let's consider the usual road traffic as an example:

O1. There are speed limits in most roads, and mobiles' speeds usually are not much higher or lower than the speed limits.

O2. In local roads, traffic signals affect mobiles' movements significantly.

O3. During the rush hours, the speeds of all mobiles in a given geographical area are closely correlated.

O4. In many cases, the direction of a mobile can be predicted from the path the mobile has taken so far.

3. This is the cell $A^{\prime}$ s (or its base station's) view.

4. Hence, we will use the terms "connection" and "mobile" interchangeably throughout this paper.
From the above observations, we expect that the hand-off behavior of a mobile will be probabilistically similar to that of the mobiles which came from the same previous cell and are now residing in the current cell. Hence, we can predict the next cell of a mobile and estimate its hand-off time by utilizing an aggregate history of observations in each cell. Even though the above observations were made from the road traffic, the same method can be used for pedestrians because the speeds of pedestrians won't differ much. In a typical outdoor cellular network, there will be both pedestrian and vehicular mobiles while in the indoor case, there are mostly pedestrians or nonmoving objects.

The location and direction of active mobiles are, in general, unknown to the underlying wired network (or BS). On the other hand, there might be a special case when the wired network knows a mobile's next cell, i.e., the cell the mobile will traverse in future. The next cell of a mobile can be either derived when the path of the mobile is known, e.g., from an ITS navigation system [21], or predicted with high accuracy when the location of the mobile is known, e.g., from the GPS. We envision that many cell phones in the future will be equipped with the GPS for the location-based and Enhanced 911 (E911) emergency services. ${ }^{5}$ How to predict the next cell of a mobile from its location information will be demonstrated with an example in Section 5. In either case, there needs to exist a communication interface between the mobile and the wired network in order to make the path or location information available to the wired network. When this nextcell information is available to the wired network, our scheme will be shown to work much better than the case without such information.

Another possibility is to use mobile-specific histories as suggested in [12]. That is, if a mobile's movements are observed over time, then the mobile's direction in a specific cell can be predicted by utilizing this observed information. However, keeping track of each mobile's mobility over time is too costly, and in many cases, mobile-specific histories are not accurate enough to make good predictions. So, we preclude the availability of such information.

\subsection{Hand-Off Estimation Functions}

We now develop a scheme to estimate and predict mobility. This scheme will be executed by the BS of each cell in a distributed manner. For each mobile which moves into an adjacent cell from the current cell 0, the cell 0's BS caches the mobile's quadruplet, $\left(T_{\text {event }}\right.$, prev, next, $\left.T_{\text {so }}\right)$, called a handoff event quadruplet, where $T_{\text {event }}$ is the time when the mobile departed from the current cell, prev is the index of the previous cell the mobile had resided in before entering the current cell, next is the index of the cell the mobile entered after departing from the current cell, and $T_{s o j}$ is the sojourn time of the mobile in the current cell, i.e., the time span between the entry into and departure from the current cell. Note that prev $=0$ means that the departed mobile started its connection in the current cell.

From the cached quadruplets, the BS builds hand-off estimation function, which describes the estimated

5. According to the Federal Communications Commission (FCC) ruling, all cell phones in the US are required to be equipped with a location device such as a GPS for E911 services in a few years [5]. 


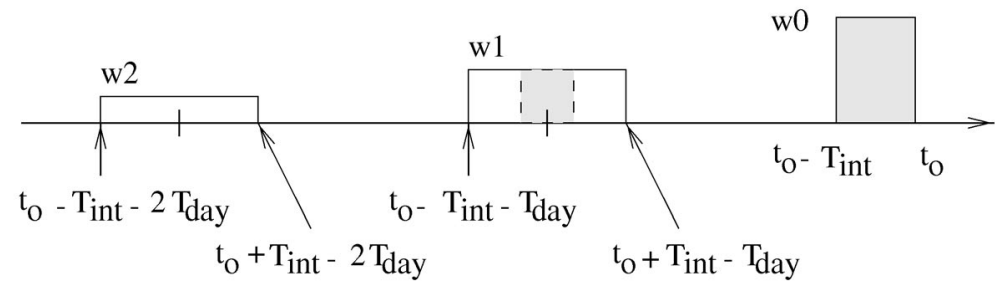

Fig. 3. An example of periodic windows to obtain hand-off estimation functions with $N_{\text {win_days }}=2$.

distribution of the next cell and sojourn time of a mobile, depending on the cell the mobile stayed before. One can also imagine that this probabilistic behavior of mobiles, especially in terms of sojourn time, will depend on the time of day, e.g., the sojourn time during rush hours will differ significantly from that during nonrush hours. We assume that the probabilistic behavior will mostly follow a cyclic pattern with the period of one day. A hand-off estimation function, at the current time $t_{o}$, is obtained as follows: for a quadruplet $\left(T_{\text {event }}\right.$, prev, next,$\left.T_{\text {soj }}\right)$ such that

$$
t_{o}-T_{\text {int }}-n T_{\text {day }} \leq T_{\text {event }}<t_{o}+T_{\text {int }}-n T_{\text {day }},
$$

where $T_{i n t}$ is the estimation interval of the function which is a design parameter, $T_{d a y}$ is the duration of a day, i.e., 24 hours, and $n(\geq 0)$ is an integer,

$$
F_{H O E}\left(t_{o}, \text { prev, next, } T_{s o j}\right):=w_{n},
$$

where $1 \geq w_{n} \geq w_{n+1}$, and $w_{n}=0$ for all $n>N_{\text {win_days. }}$. The weight factor $w_{n}$ is from the fact that the traffic condition in a cell during a specific period of days can vary over time. $N_{\text {win_days }}$ is a design parameter so that the quadruplet observed more than $\left(N_{\text {win_days }} \cdot T_{d a y}+T_{\text {int }}\right)$ ago is determined out-of-date, and not used for the hand-off estimation function. One can easily see that the hand-off estimation functions are affected by the hand-off event quadruplets within the periodic windows of duration $2 T_{i n t}$ as shown in Fig. 3. Note that the duration $\left[t_{o}, t_{o}+T_{i n t}\right]$ is missing in the figure because it represents a future time, which is not meaningful in the definition of a hand-off event quadruplet.

In practice, it is desirable to limit the number of the quadruplets 1) used for the hand-off estimation function and 2) currently not used for the hand-off estimation function, but cached for future use, e.g., those with $t_{o}+T_{\text {int }}-T_{\text {day }}<$ $T_{\text {event }}<t_{o}-T_{\text {int }}$ in Fig. 3, in order to reduce the memory and computation complexity. ${ }^{6}$ We define the maximum handoff estimation function size, $N_{\text {quad }}$, as the maximum number of hand-off event quadruplets used for the hand-off estimation function for each prev. This implies that we don't need the quadruplets from previous days if we observed enough during the last $T_{\text {int }}$ interval. Up to $N_{\text {quad }}$ cached quadruplets are used for the hand-off estimation with the following priority rule. First, the quadruplet which satisfies (2) with a smaller $n$ gets higher priority. Second, among those satisfying (2) with the same $n$, the quadruplet with a smaller $\mid T_{\text {event }}-$ $n T_{\text {day }}$ / gets higher priority. Fig. 3 shows an example that only the quadruplets with the event times $T_{\text {event }}$ within the shaded

6. The calculations for the mobility estimation will be dependent on the number of the quadruplets used for the hand-off estimation function as will be shown in the next section. regions are used for the hand-off estimation function according to the priority rule, implying that the total number of quadruplets within the regions is $N_{\text {quad }}$. In order to reduce the cache memory size, those quadruplets observed at time $t^{\prime}$, i.e., $T_{\text {event }}=t^{\prime}$, when the hand-off estimation function at time $t^{\prime}$ doesn't use any quadruplets observed previous days are not cached for future use, because they are unlikely to be used for the hand-off estimation function next day. Note that those quadruplets 1) with $T_{\text {event }}<t_{o}-T_{\text {int }}-N_{\text {win_days }} T_{\text {day }}$ and 2) not used for the hand-off estimation function during the last $\left(T_{d a y}+T_{\text {int }}\right)$ can be deleted from the cache memory.

Fig. 4 shows an example of footprint of the hand-off estimation function for $p r e v=1$ without showing the values of $w_{n}$ 's. The number of dots in the footprint, each of which corresponds to a cached hand-off event quadruplet, will be bounded by $N_{\text {quad }}$. In the hand-off estimation function in a 3-dimensional space, the function is shown to have different heights, depending on the values of $w_{n}$ 's. The example is drawn from the same indexing as shown in Fig. 2b. From the footprint, we observe that cell 4 is the farthest cell from cell 1 (i.e., the previous cell) through cell 0 (i.e., the current cell) among the adjacent cells of cell 0 since the sojourn times before entering cell 4 are generally shown to be among the largest. Note that the hand-off estimation function for a given prev can generate a probability mass function for a two-dimensional random vector $\left(\right.$ next, $\left.T_{s o j}\right)$, where next is the predicted next cell and $T_{s o j}$ is the estimated sojourn time in the current cell. How this handoff estimation function is used to estimate the user mobility is discussed next.

\section{Predictive, Adaptive Bandwidth Reservation and Admission Control}

We now describe predictive, adaptive bandwidth reservation and admission control to keep the hand-off dropping probability $P_{H D}$ below $P_{H D \text {,target }}$ by utilizing the hand-off estimation functions described thus far.

\subsection{Bandwidth Reservation without Next-Cell Information}

The scheme considered here is for the general case when the wired network (or BS) does not have the next-cell information of mobiles. The special case when BSs have the next-cell information will be treated in the next section. Our approach is based on the estimated mobility during the time window $\left[t_{o}, t_{o}+T_{e s t}\right]$, where $t_{o}$ is the current time. We consider the behavior of a mobile in the current cell. The mobility of the active mobile with connection $C_{0, j}$ is 


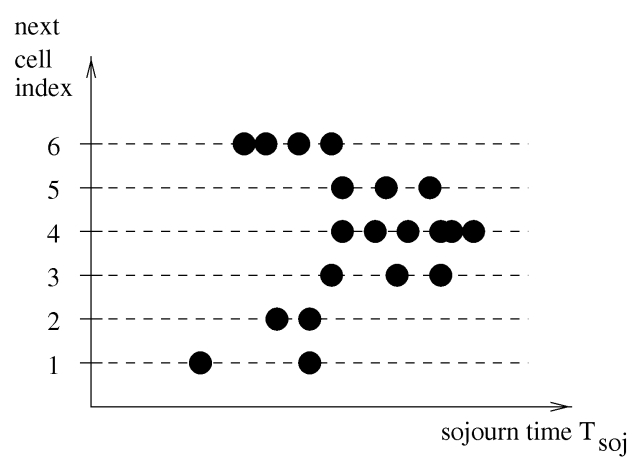

Fig. 4. An example of the footprint of the hand-off estimation function for prev $=1$.

estimated with $p_{h}\left(C_{0, j} \rightarrow i\right)$, the probability that $C_{0, j}$ hands off into cell $i$ within $T_{\text {est }}$.

The hand-off probability can be computed using the hand-off estimation function as follows: The BS of a cell keeps track of each active mobile in its cell via the mobile's extant sojourn time. The extant sojourn time $T_{\text {ext_soj }}\left(C_{0, j}\right)$ of connection $C_{0, j}$ is the time elapsed since the active mobile with connection $C_{0, j}$ entered the current cell. Using Bayes' theorem [16], the hand-off probability $p_{h}\left(C_{0, j} \rightarrow\right.$ next $)$ at time $t_{o}$ is calculated by

$$
\begin{aligned}
& p_{h}\left(C_{0, j} \rightarrow \text { next }\right):=
\end{aligned}
$$

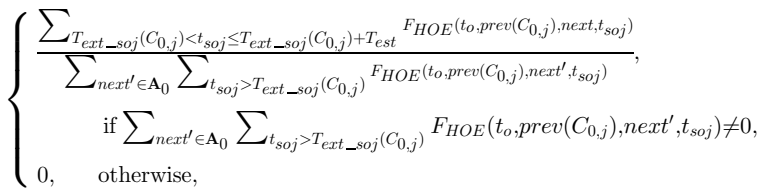

in which $\operatorname{prev}\left(C_{0, j}\right)$ is the cell which $C_{0, j}$ resided in before entering the current cell and $\mathbf{A}_{i}$ is the set of indices of cell $i^{\prime}$ s adjacent cells. The equation represents the expected probability that $C_{0, j}$ hands off into cell next with the sojourn time $t_{s o j}$ which is less than, or equal to, $T_{\text {ext_soj }}\left(C_{0, j}\right)+T_{\text {est }}$ given the condition that $t_{s o j}>T_{e x t_{-} s o j}\left(C_{0, j}\right)$, which is the hand-off probability $p_{h}\left(C_{0, j} \rightarrow\right.$ next $)$.

Fig. 5a shows an example of calculating $p_{h}\left(C_{0, j} \rightarrow 4\right)$, when $C_{0, j}$ entered cell 0 from cell 1 , using the footprint of

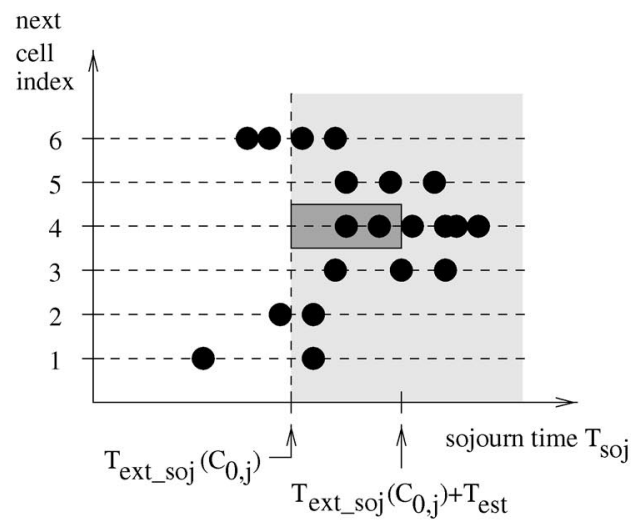

(a) the hand-off estimation function for $\operatorname{prev}\left(C_{0, j}\right)=1$, shown in Fig. 4. In the figure, the values of $F_{H O E}\left(t_{o}, 1, n e x t^{\prime}, T_{s o j}\right)$ from all points at the right side of the vertical line at $T_{s o j}=$ $T_{\text {ext_soj }}\left(C_{0, j}\right)$ (i.e., in both dark and light shaded regions) are summed to obtain the denominator in (4). Because this value is not zero, the values of $F_{H O E}\left(t_{o}, 1,4, T_{s o j}\right)$ from two points in the dark-shaded region are summed to obtain the numerator in (4). Then, we can complete the calculation of $p_{h}\left(C_{0, j} \rightarrow 4\right)$. Note that the mobile with connection $C_{0, j}$ is estimated to be stationary (i.e., nonmoving) in cell 0 if there is no hand-off event in the hand-off estimation function with a sojourn time larger than the connection $C_{0, j}$ 's extant sojourn time, i.e., the denominator in (4) is zero.

Now, using the probabilities of handing off connections into cell 0 from its adjacent cell $i$ within $T_{\text {est }}$ (i.e., hand-off probabilities $p_{h}\left(C_{i, j} \rightarrow 0\right)$ ), the required bandwidth $B_{r 0}^{i}$ to be reserved in cell 0 for the expected hand-offs from cell $i$ is obtained as:

$$
B_{r, 0}^{i}=\sum_{j \in \mathbf{C}_{i}} b\left(C_{i, j}\right) p_{h}\left(C_{i, j} \rightarrow 0\right),
$$

where $\mathbf{C}_{i}$ is the set of indices of the connections in cell $i$ and $b\left(C_{i, j}\right)$ is connection $C_{i, j}$ 's bandwidth. Finally, the target reservation bandwidth $B_{r, 0}$ in cell 0 , which is the aggregate bandwidth to be reserved in cell 0 for the expected handoffs from adjacent cells within $T_{e s t}$, is calculated as:

$$
B_{r, 0}=\sum_{i \in \mathbf{A}_{0}} B_{r, 0}^{i},
$$

where $\mathbf{A}_{i}$ is the set of indices of cell is neighbors.

Note that the target reservation bandwidth is an increasing function of the estimation time $T_{e s t}$ as $p_{h}\left(C_{i, j} \rightarrow 0\right)$ is an increasing function of $T_{\text {est }}$. There might be an optimal value of $T_{e s t}$ for given traffic load and user mobility that minimizes the new connection blocking probability while keeping the handoff dropping probability below the target. In the proposed scheme, $T_{\text {est }}$ will be adjusted adaptively in each cell independently of others, depending on the hand-off dropping events observed in the cell as described in Section 4.3. The estimation time $T_{\text {est }}$ of cell next (or $T_{\text {est,next }}$ ) will then be used in (4). So, when the BS of cell 0 needs to update the value

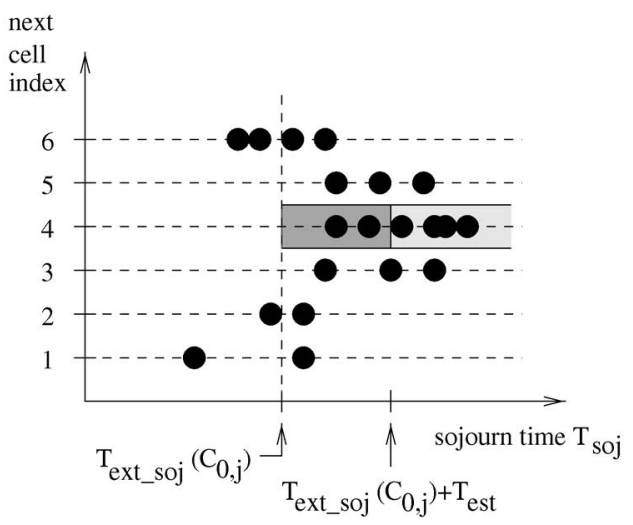

(b)

Fig. 5. An example of calculating $p_{h}\left(C_{0, j} \rightarrow\right.$ next $)$ when $\operatorname{prev}\left(C_{0, j}\right)=1$ and next $=4$ using the footprint of $F_{H O E}\left(t_{o}, \operatorname{prev}\left(C_{0, j}\right), n e x t^{\prime}, T_{s o j}\right)$. (a) Without next-cell information. (b) With next-cell information. 
of $B_{r, 0}$, the BS will inform its adjacent cells of the current value of $T_{e s t, 0}$ and then the BS of each adjacent cell will calculate the required bandwidth for the expected hand-offs from that cell-that is, $B_{r, 0}^{i}$ for cell $i$ - using (5), and will inform cell 0 's BS of this value. Finally, cell 0's BS will calculate $B_{r, 0}$ using (6).

\subsection{Bandwidth Reservation with Next-Cell Information}

The reservation scheme described in the previous section is modified to utilize the next-cell information of a mobile, if available. This special case can happen, for example, when a mobile user is driving a car equipped with a navigation system, which guides the user to a specified destination from the current location using the GPS and an ITS route guidance system, and the navigation system informs the current cell's BS of the next cell the user is supposed to move to. On the other hand, the mobile's next cell can also be predicted with high accuracy when its location and direction are known to the wired network. This becomes possible, for example, when a mobile is equipped with the GPS, like a GPS-enabled cell phone for the location-based and E911 emergency services, and the mobile informs its current cell's BS of its location and direction. How to predict the mobile's next cell from its location and direction information is demonstrated in Section 4.3. For ease of explanation, however, we assume that the perfect knowledge of a mobile's next-cell information is available to the wired network here.

In the previous section, a BS utilized the hand-off estimation functions for two purposes: one is to predict the next cell a mobile will move to, and the other is to estimate the mobile's sojourn time in the current cell. With a mobile's next-cell information, the hand-off estimation function needs to be used solely for the second purpose. Suppose the mobile with connection $C_{0, j}$ in the current cell will leave for cell next, then

$$
p_{h}\left(C_{0, j} \rightarrow n e x t^{\prime}\right):=0, \quad \text { if } n e x t^{\prime} \neq n e x t,
$$

and (4\}) is modified to: ${ }^{7}$

$$
\begin{aligned}
& p_{h}\left(C_{0, j} \rightarrow \text { next }\right):=
\end{aligned}
$$

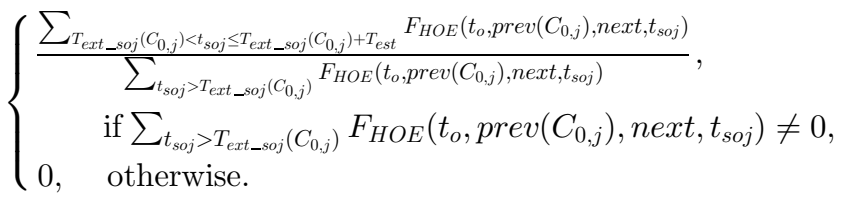

Fig. $5 \mathrm{~b}$ shows an example of calculating $p_{h}\left(C_{0, j} \rightarrow 4\right)$ with next-cell information, when connection $C_{0, j}$ came from cell 1 , using the footprint of the hand-off estimation function for $\operatorname{prev}\left(C_{0, j}\right)=1$, shown in Fig. 4 . In the figure, the values of $F_{H O E}\left(t_{o}, 1,4, T_{s o j}\right)$ from the points in both dark and light

7. Logically, one may think that $p_{h}\left(C_{0, j} \rightarrow\right.$ next $)$ should be assigned 1 instead of 0 in the "otherwise" case because the extant sojourn time is larger than the maximum sojourn time in the mobility estimation function, meaning that the mobile will soon move into cell next. In practice, the maximum sojourn time might not be so reliable as to represent the real maximum sojourn time since only a finite number of hand-off event quadruplets are used in the mobility estimation function and the possible maximum sojourn time itself is time-varying. Assigning 1.0 to $p_{h}\left(C_{0, j} \rightarrow\right.$ next) was found to result in frequent over-reservations. shaded regions are summed to obtain the denominator in (8). Because this value is not zero, the values of $F_{H O E}\left(t_{o}, 1,4, T_{s o j}\right)$ from two points in the dark shaded region are summed to obtain the numerator in (8). Then, the value of $p_{h}\left(C_{0, j} \rightarrow 4\right)$ is obtained. Now, the hand-off probabilities using (7) and (8) are used in (5) and (6) to calculate the target reservation bandwidth. This modified scheme is expected to outperform the previous scheme in terms of estimation accuracy and, hence, bandwidthreservation efficiency.

\subsection{Control of Mobility Estimation Time Window}

Using the proposed scheme, the bandwidth will be overreserved (underreserved) for hand-offs if $T_{\text {est }}$ is too large (small). There might exist an optimal value of $T_{\text {est }}$ for given traffic load and user mobility, but these parameters, in practice, vary with time. Moreover, the mobility estimation functions used may not reflect mobiles' behaviors well, thus resulting in inaccurate mobility estimation even with the optimal $T_{\text {est }}$. We therefore propose an adaptive algorithm for controlling the mobility estimation time window based on the hand-off dropping events observed in each cell so as to approximate the optimal $T_{\text {est }}$ over time. Fig. 6 shows the algorithm executed by the BS in each cell to adjust the value of $T_{\text {est }}$.

Before running the algorithm, the reference window size $w\left(=\left\lceil 1 / P_{H D, \text { target }}\right\rceil\right)$ is determined and assigned to the observation window size $w_{o b s}$. In addition, $T_{e s t}$ is initialized to $T_{\text {start }}$, a design parameter, and the counts for hand-offs $n_{H}$ and hand-off drops $n_{H D}$ are reset to 0 . As can be found in the pseudo-code, $w_{o b s}$ is increased or decreased by $w$, and the constraint $P_{H D}<P_{H D \text {,target }}$ can be translated into that of keeping the counted number $n_{H D}$ of hand-off drops out of a total of $w_{o b s}$ observed hand-offs below $w_{o b s} / w$. During the runtime, whenever there is a hand-off drop after $w_{\text {obs }} / w$ drops, $T_{\text {est }}:=T_{\text {est }}+1$ and $w_{o b s}:=w_{o b s}+w$. On the other hand, when there were less than, or equal to, $w_{o b s} / w$ handoff drops out of $w_{o b s}$ observed hand-offs, $T_{e s t}:=T_{e s t}-1$ and $w_{o b s}:=w . T_{e s t}$ is not greater than $T_{s o j, \max }$ in Fig. 6, which is the maximum $T_{s o j}$ derived from the hand-off estimation functions in adjacent cells, because any value larger than that is meaningless. We also set the minimum value of $T_{e s t}$ to 1 since if the value is too small, our scheme will reserve virtually no bandwidth, irrespective of the existing connections in adjacent cells.

Given below are some considerations for the design of the estimation time window control algorithm.

C1. When there were more hand-off drops than permitted, the algorithm should start to increase $T_{\text {est }}$ quickly because of the underreserved bandwidth; otherwise, there will be continued hand-off drops.

$\mathrm{C} 2$. The increment of $T_{\text {est }}$ should not be too high. Otherwise, it might result in an overreaction, hence overreservation.

C3. Due to overreaction or decreased traffic load over time, there might be fewer hand-off drops than permitted, so the value of $T_{\text {est }}$ should be decreased quickly. Otherwise, the bandwidth will continue to be overreserved, hence underutilizing the system. 


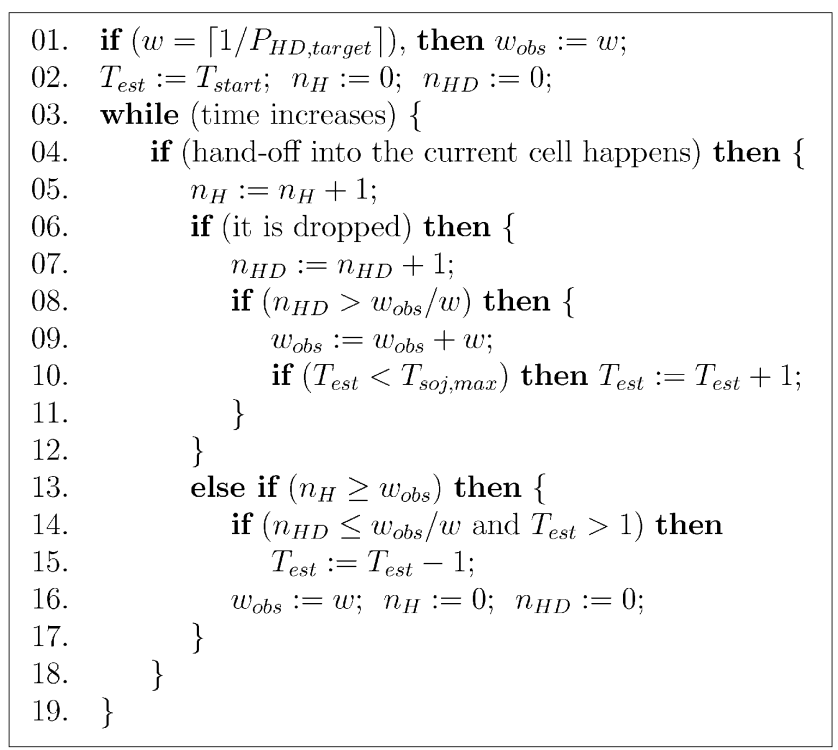

Fig. 6. A pseudo-code of the algorithm to adjust $T_{\text {est }}$ in each BS.

C4. $T_{\text {est }}$ should not be decreased too much. Otherwise, it might result in an overreaction, hence underreservation.

There can be many candidate algorithms satisfying the above requirements. Especially, there might be many choices of increment and decrement step sizes, both of which were fixed at 1 . We experimented with other choices like additive and multiplicative step sizes: the step size was increased additively $(1,2,3, \cdots)$ or multiplicatively $(1,2,4, \cdots)$ for consecutive increments and decrements. The main purpose of these choices is a prompt reaction to hand-off drops, i.e., C1 and C3. However, these choices are found to cause overreactions, and make the reserved bandwidth fluctuate severely between overreservation and underreservation. The algorithm presented here is the best one we have found so far.

\subsection{Admission Control}

The admission test after calculating the target reservation bandwidth can be as simple as given in (1). That is,

1. Check if $\sum_{j \in \mathbf{C}_{0}} b\left(C_{0, j}\right)+b_{\text {new }} \leq C(0)-B_{r, 0}$.

2. If the above test is positive, the connection is admitted,

where $C(0)$ and $b_{\text {new }}$ are the link capacity of cell 0 and the bandwidth of the newly-requested connection, respectively. This simple admission-control scheme will henceforth be referred to as AC1. However, when there is not enough bandwidth left unused by existing connections that can be reserved for hand-offs, it is meaningless to calculate the target reservation bandwidth. If this situation lasts for an extended period due to continued incoming hand-offs, the problem becomes more serious because some of the incoming hand-offs will be continuously dropped due to the unavailability of reserved bandwidth, triggering further increase of $T_{\text {est }}$. This, in turn, requires to reserve more bandwidth that doesn't exist. This situation can happen when adjacent cells accept new connections solely based on the admission test using (1) and those admitted connections continue to be handed off into the current cell even though it doesn't have enough bandwidth.

To handle this problem, the admission test should check available bandwidths of adjacent cells as well as the current cell. More precisely, one must check if there is enough bandwidth in the current and next cell of each newlyrequested connection, but, without next-cell information, it is not possible to know the next cell. Thus, depending on whether next-cell information is available or not, we apply different admission control schemes. First, without next-cell information:

1. For all $i \in \mathbf{A}_{0}$, check if $\sum_{j \in \mathbf{C}_{i}} b\left(C_{i, j}\right) \leq C(i)-B_{r, i}$,

2. Check if $\sum_{j \in \mathbf{C}_{0}} b\left(C_{0, j}\right)+b_{\text {new }} \leq C(0)-B_{r, 0}$,

3. If all of the above tests are positive, then the connection is admitted.

We call this scheme AC2. Second, with next-cell information, i.e., we know that the new connection will later move to cell next:

1. If $n e x t \neq 0$, then check if

$$
\sum_{j \in \mathbf{C}_{\text {next }}} b\left(C_{n e x t, j}\right) \leq C(\text { next })-B_{r, n e x t},
$$

2. Check if $\sum_{j \in \mathbf{C}_{i}} b\left(C_{i, j}\right)+b_{\text {new }} \leq C(0)-B_{r, 0}$,

3. If the above two tests are positive, then the connection is admitted.

This scheme is referred to as AC2 w/ NC. Note that next could be 0 , and in this case, the first step can be skipped.

Note that using the above admission test in the absence of a mobile's next-cell information, the current cell and all of its adjacent cells must calculate $B_{r, i}$ for each new admission request, and this is costly. In fact, the undesirable situation described in the beginning of this section is expected to happen only in heavily-loaded networks. So, we present a hybrid scheme which requires only those adjacent cells which "appear" to be unable to reserve the target reservation bandwidth, to calculate the target bandwidth again and participate in the admission test. Note that $B_{r, i}$ is a timevarying function, and updated upon admission test. Upon arrival of a new connection request at cell 0 , if the current target reservation bandwidth of an adjacent cell $i, B_{r, i}^{\text {curr }}$, which was calculated for a previous admission test, is not reserved fully, this cell will recalculate $B_{r, i}$, and participate in the admission test. First, without next-cell information (AC3):

1. For all $i \in \mathbf{A}_{0}$ such that $\sum_{j \in \mathbf{C}_{i}} b\left(C_{i, j}\right)+B_{r, i}^{\text {curr }}>C(i)$, check if $\sum_{j \in \mathbf{C}_{i}} b\left(C_{i, j}\right) \leq C(i)-B_{r, i}$

2. Check if $\sum_{j \in \mathbf{C}_{0}} b\left(C_{0, j}\right)+b_{\text {new }} \leq C(0)-B_{r, 0}$,

3. If all the above tests are positive, then the connection is admitted.

Second, with next-cell information, or when the newlyrequested connection will later depart to cell next (AC3 w/ NC):

1. If next $\neq 0$ and $\sum_{j \in \mathbf{C}_{\text {next }}} b\left(C_{\text {next }, j}\right)+B_{r, \text { next }}^{\text {curr }}>C($ next $)$,

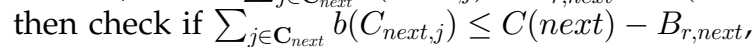

2. Check if $\sum_{j \in \mathbf{C}_{i}} b\left(C_{i, j}\right)+b_{\text {new }} \leq C(0)-B_{r, 0}$, 
TABLE 1

Summary of the Admission-Control Schemes

\begin{tabular}{|l|c|c|}
\hline \multicolumn{1}{|c|}{ Name } & Next-Cell Information & Description \\
\hline \hline AC1 & without & Calculation of $B_{r}$ in the current cell only. \\
\hline AC2 & without & $\begin{array}{c}\text { Calculation of } B_{r} \text { in the current cell } \\
\text { and every adjacent cell. }\end{array}$ \\
\hline AC2 w/ NC & with & $\begin{array}{c}\text { Calculation of } B_{r} \text { in the current cell } \\
\text { and some adjacent cells only. }\end{array}$ \\
\hline AC3 & without & with \\
\cline { 1 - 2 } AC3 NC & and &
\end{tabular}

3. If the above two tests are positive, then the connection is admitted.

Table 1 shows the summary of the admission-control schemes described above. These schemes will be comparatively evaluated in the next section.

\section{Comparative Performance Evaluation}

This section presents and discusses the evaluation results of the proposed schemes as well as the static reservation scheme for comparative purposes. We first describe the assumptions and specifications used for the simulation study.

\subsection{Simulation Assumptions and Specifications}

We consider two different simulation environments: onedimensional and two-dimensional cases. For the onedimensional environment, mobiles travel along a straight road (e.g., cars on a highway). This environment is the simplest in the real world, representing a one-dimensional cellular system as in Fig. 2a. We make the following assumptions for our simulation study:

A1. The whole cellular system is composed of 10 linearlyarranged cells, for which the diameter of each cell is $1 \mathrm{~km}$. Cells are numbered from 1 to 10 , i.e., cell $\langle i\rangle$ represents the $i$ th cell.

A2. Connection requests are generated according to a Poisson process with rate $\lambda$ (connections/second/ cell) in each cell. A newly-generated connection can appear anywhere in the cell with an equal probability.

A3. A connection is either for voice (requiring $1 \mathrm{BU}$ ) or for video (requiring 4 BUs) with probabilities $R_{v o}$ and $1-R_{v o}$, respectively, where the voice ratio $R_{v o} \leq 1$.

A4. Mobiles can travel in either of two directions with an equal probability with a speed chosen randomly between $S P_{\min }$ and $S P_{\max }(\mathrm{km} /$ hour$)$. Each mobile will run straight through the road with the chosen speed, i.e., mobiles will never turn around.

A5. Each connection's lifetime is exponentially-distributed with mean 120 (seconds).

A6. Each cell has a fixed link capacity 100 BUs, i.e., $C(i)=C=100$ for all $i$.

Note that the fixed capacity assumption does not necessarily hold in practice. For example, CDMA systems have a softer notion of capacity, in which the capacity depends on the target interference level. This target interference level is affected by the desired error performance of the system, which can be negotiable in some case.

For the two-dimensional environment, the roads are arranged in a mesh shape, and a BS is located at each intersection of two crossing roads as shown in Fig. 7. This cellular structure can typically be seen in a metropolitan downtown area. We make the following assumptions for this two-dimensional environment:

B1. The cellular system is composed of 25 cells (i.e., a $5 \times 5$ mesh), and each cell's diameter is $300 \mathrm{~m}$.

B2. Mobiles can travel in either of two directions along a road with an equal probability at a speed chosen randomly within the range $[40,60]$ ( $\mathrm{km} /$ hour).

B3. At the intersection of two roads, a mobile might continue to go straight, or turn left, right, or around with probabilities $0.55,0.2,0.2$, and 0.05 , respectively.

B4. If a mobile chooses to go straight or turn right at the center of a cell, it might need to stop there with probability 0.5 for a random time between 0 and 30 (secs) due to a red traffic light.

B5. If a mobile chooses to turn left or around, it needs to stop there for a random time between 0 and 60 (secs) due to the traffic signal.

B6. The link capacity $C$ is 50 BUs.

B7. The assumptions A2, A3, and A5 above are also made.

The rationale behind the assumed mobile's delay at the intersection is that there are four traffic signals at the intersection for mobiles arriving from the four directions, respectively. A traffic signal will have the red (for stop), leftturn, green (for going straight and turning right) lights in order, then returning to the red light. The whole period from red light to the next red is $60+\epsilon$ seconds in which the red light will last for 30 seconds, then the left-turn light will turn on for a very short time $\epsilon$, then, finally, the green light will last for 30 seconds.

Each simulation run starts without any prememorized hand-off event quadruplets. As simulations are run, quad-

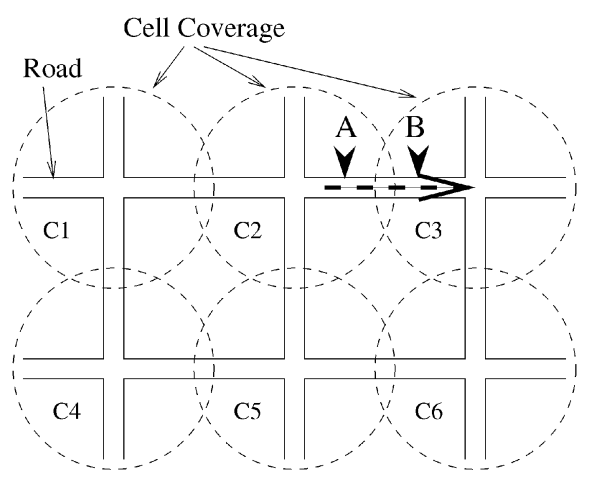

Fig. 7. A cellular structure. 


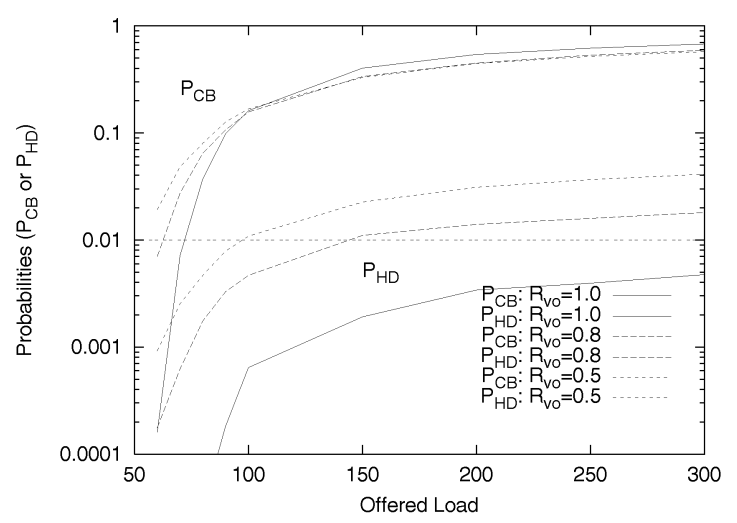

(a)

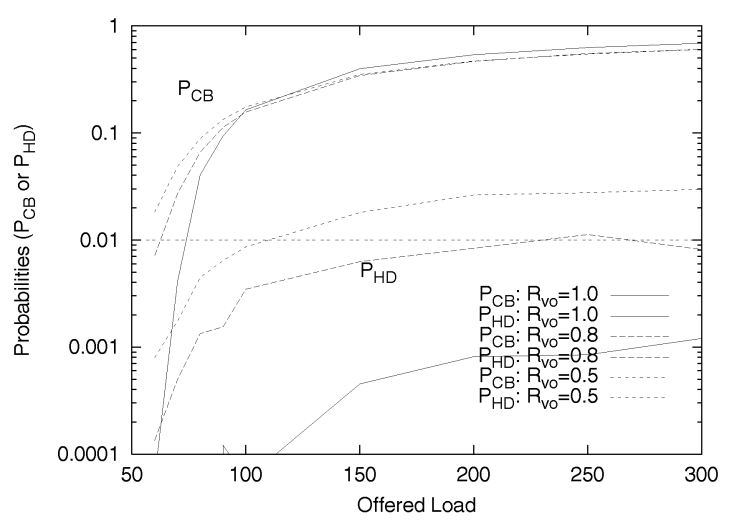

(b)

Fig. 8. $P_{C B}$ and $P_{H D}$ versus offered load: static reservation with $B_{r}=10 \mathrm{BUs}$. (a) High user mobility. (b) Low user mobility.

ruplets will be collected, and will affect the hand-off estimation functions $F_{H O E}\left(t, p r e v, n e x t, T_{s o j}\right)$. Under the above assumptions, the border cells (i.e., cells $<1>$ and $<$ $10>$ ) will face fewer mobiles because there are no mobiles entering from the outside of the cellular system. Then, cells near the center (such as cells $<5>$ and $<6>$ ) will be more crowded by mobiles than those near the borders. This uneven traffic load can affect the performance evaluation of our proposed schemes, hence making it difficult to assess their operations correctly. So, we connected two border cells, i.e., cells $\langle 1\rangle$ to $\langle 10\rangle$, artificially so that the whole cellular system forms a ring architecture as was assumed in [14] (unless stated otherwise). With the same reasoning, two end roads in the border cells at the boundary of the cellular structure are also connected in the two-dimensional case. For example, in Fig. 7 , the left-most (upper-most) road in cell $C 1$ is connected to the right-most (lower-most) road in cell $C 3(C 4)$.

We specifically use the two-dimensional case for the comparison between the schemes with and without the nextcell information as the effectiveness of the next-cell information will not be evident in the one-dimensional environments. The one-dimensional case is assumed unless stated otherwise. The parameters used include: $P_{H D \text {,target }}=0.01, T_{\text {start }}=$ 1 (second), $N_{\text {quad }}=100, T_{\text {int }}=1$ (hour), $N_{\text {win_days }}=1$, and $w_{0}=w_{1}=1$. A frequently-used measure is the offered load per cell, $L$, which is defined as connection generation rate $\times$ connections' bandwidth $\times$ average connection lifetime, i.e.,

$$
L=\left(1 \cdot R_{v o}+4 \cdot\left(R_{v o}-1\right)\right) \cdot \lambda \cdot 120,
$$

with the above-described assumptions. The physical meaning of the offered load per cell is the total bandwidth required on average to support all existing connections in a cell.

For the one-dimensional case, we considered a range of the offered load from 60 to 300. Generally, the desirable range of the offered load is less than, or equal to, the link capacity, 100 BUs, of each cell. It is undesirable to keep a cell overloaded (i.e., the offered load is $>100$ ) for an extended period of time, and in such a case, the cell must be split into multiple cells to increase the total system capacity. However, cells can get overloaded temporarily. Suppose a mobile user's connection request is blocked once. Then, she/he is expected in most cases to continue to request a connection establishment until it is successful or she/he gives up. This likely behavior of mobile users will affect the offered load. Near the offered load $=100, P_{C B}$ will be around, or larger than, 0.1 in most cases, due to some reserved bandwidth for hand-offs, and in such a situation, if each connection-blocked user attempts to make a connection about five times, then the offered load will increase to about 150 in a very short time. Likewise, there might be some cases with the offered load of 300 . This possible situation can be interpreted as a positive-feedback effect for increase in the offered load. We consider the large values of offered load such as 300 , since even for these large offered loads, our goal to keep $P_{H D}$ below a target value should be achieved. With a similar reasoning, we consider a range of the offered load from 20 to 100 for the two-dimensional case, where the link capacity is 50 BUs.

\subsection{Stationary Traffic/Mobility}

First, we simulated for stationary traffic/mobility with constant new connection generation rate $\lambda$ and mobile speed range $\left[S P_{\min }, S P_{\max }\right]$. Two cases of user mobility are considered: high user mobility with $S P_{\min }=80$ \& $S P_{\max }=120$, and low user mobility with $S P_{\min }=40 \&$ $S P_{\max }=60$. For the stationary case, $T_{\text {int }}=\infty$ is used since the speed range and the offered load do not vary during each simulation run; so, $N_{\text {days_win }}=1$ is meaningless.

\subsubsection{Static Reservation}

First, we consider the performance of static reservation scheme [7], in which a portion of the link capacity is permanently reserved for hand-offs, as a reference (for comparison). Fig. 8 plotted $P_{C B}$ and $P_{H D}$ as the offered load increases for Fig. 8a high user mobility and Fig. 8b low user mobility when $B_{r}=10$, i.e., 10 BUs are reserved permanently for hand-offs in each cell. Three different values of the voice ratio $R_{v o}$ are examined: $R_{v o}=1.0,0.8$, and 0.5 . The performance of this static scheme, in terms of both probabilities, is found to depend heavily on the voice ratio, user mobility, and offered load. Examples are:

1. Static reservation of $10 \mathrm{BUs}$ suffices to achieve the design goal for $R_{v o}=1.0$, but is not enough for $R_{v o}=0.5$. 


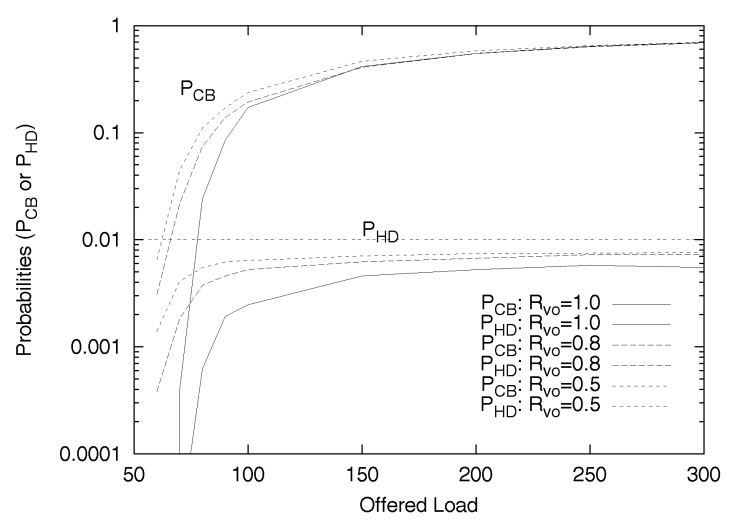

(a)

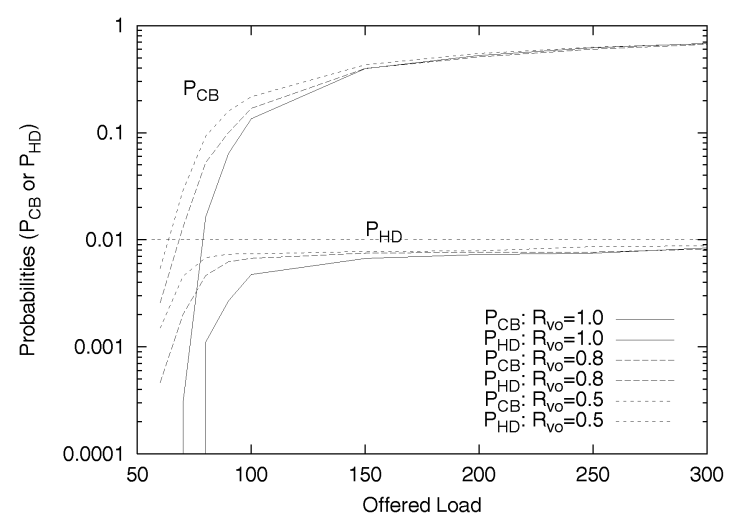

(b)

Fig. 9. $P_{C B}$ and $P_{H D}$ versus offered load: AC3. (a) High user mobility.

(b) Low user mobility.

2. For $R_{v o}=0.8,10-\mathrm{BU}$ reservation seems enough for low user mobility as shown in Fig. 8b, but not enough for high user mobility as shown in Fig. 8a.

3. For $R_{v o}=0.8$ and high user mobility, 10-BU reservation seems not enough for a highly overloaded case (i.e., $L>150$ ), but enough for the other case (i.e., $L<150)$. Moreover, for $R_{v o}=1.0,10$-BU reservation seems more than enough (i.e., overreserved) for the underloaded case (i.e., $L<100)$ since the observed $P_{H D}$ value is too small $(<0.001$ for high user mobility, and $<0.0001$ for low user mobility), compared to $P_{H D \text {,target }}=0.01$.

The voice ratio, mobile user speed, and offered load could in reality be any value and can even fluctuate. Hence, our goal cannot be achieved with static reservation, necessitating some form of adaptive reservation.

\subsubsection{Adaptive Reservation without Next-Cell Information}

First, we consider the performance of AC3, which is claimed to be the best without next-cell information among the three alternatives. Fig. 9 shows $P_{C B}$ and $P_{H D}$ as the offered load increases for Fig. 9a high user mobility and Fig. $9 \mathrm{~b}$ low user mobility. For the entire range of the offered

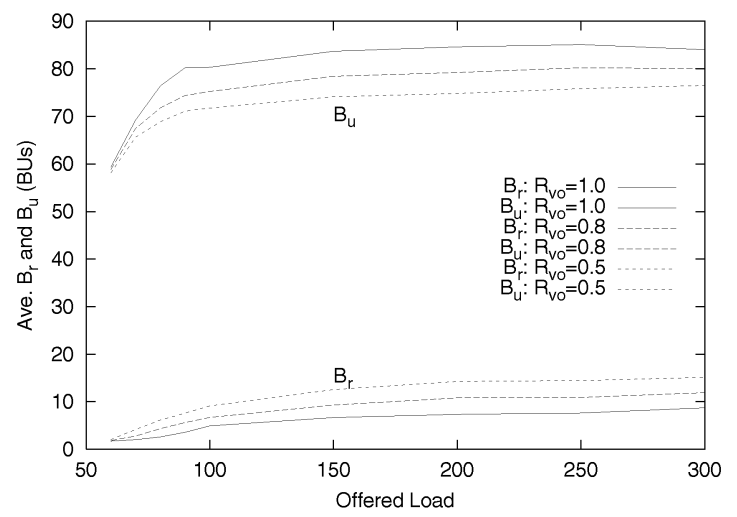

(a) load we examined, $P_{H D}$ is observed to be less than, or equal to, our target $P_{H D \text {,target }}(=0.01)$ irrespective of user mobility and voice ratio. Moreover, for given user mobility and voice ratio, the difference between $P_{C B}$ and $P_{H D}$ in the plot (of log scale) is getting smaller as the offered load decreases. This means that, as the offered load decreases, the BSs reserve less bandwidth. This is desirable as long as $P_{H D}$ stays below the target value as shown in the graphs.

Adaptive reservation patterns while varying the offered load are plotted in Fig. 10 with the average target reservation bandwidth $B_{r}$ in each cell and the average bandwidth $B_{u}$ used by the existing connections in each cell. As the offered load increases, $B_{r}$ in a cell increases monotonically, meaning that the target reservation bandwidth is controlled based on the offered load. The target reservation bandwidth gets saturated at the overloaded region, because for the entire overloaded region, regardless of the exact offered load value, the number of establishable connections will be limited by the link capacity. Our adaptive scheme reserves the bandwidth depending on the existing connections in adjacent cells and, hence, the amount of the target reservation bandwidth will be almost the same for the entire overloaded region.

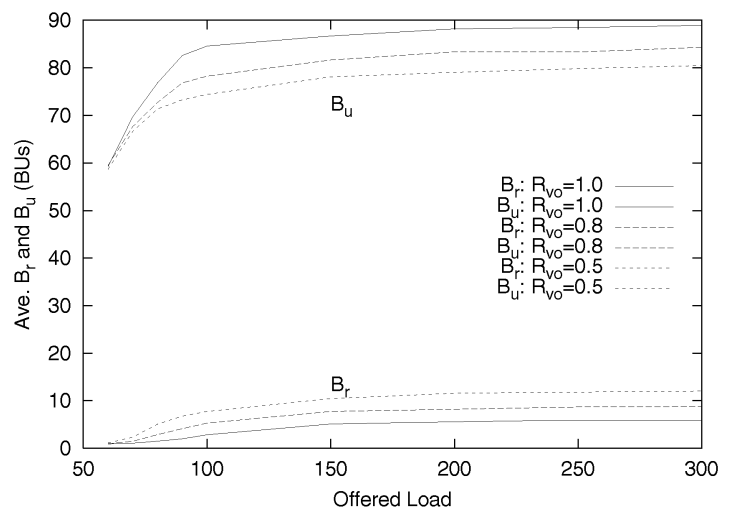

(b)

Fig. 10 Average target reservation bandwidth $B_{r}$ and average bandwidth used $B_{u}$ versus offered load: AC3. (a) High user mobility. (b) Low user mobility. 


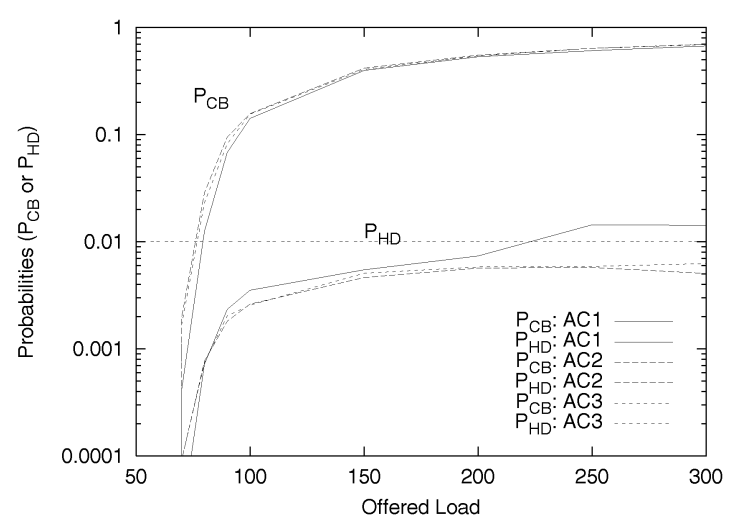

(a)

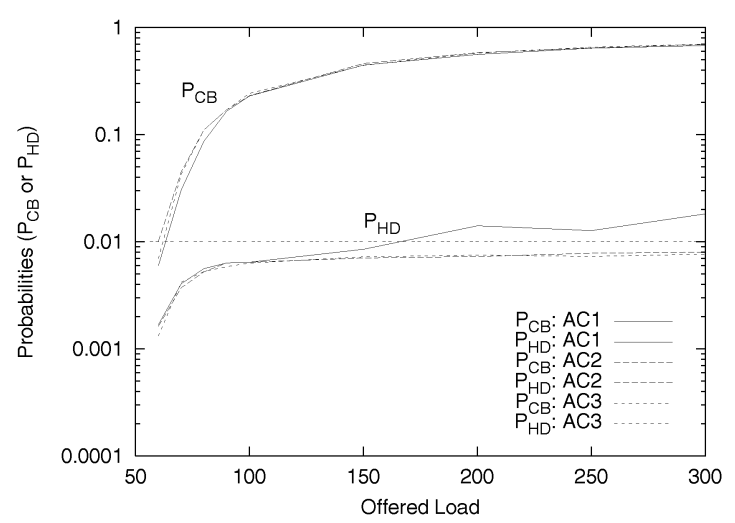

(b)

Fig. 11. Comparison among AC1, AC2, and AC3 using $P_{C B}$ and $P_{H D}$ versus offered load for high user mobility. (a) $R_{v 0}=1.0$. (b) $R_{v 0}=5.0$.

We also observe that the target reservation bandwidth increases as the voice ratio $R_{v o}$ decreases since the more video connections exist, the more bandwidth is needed. The average bandwidth used $B_{u}$ is inversely proportional to the average target reservation bandwidth $B_{r}$ since the reserved bandwidth can be used for handed-offs only. The reason why the sum of $B_{u}$ and $B_{r}$ is less than the capacity, 100 , is that in AC3, the reserved bandwidths in adjacent cells are also checked for the admission test when these cells are suspected to have been overloaded. By comparing two user mobility cases, we observe that, for similar offered load and voice ratio, the high-mobility case reserves more bandwidth than the low-mobility case. For the low-mobility case, the chance of hand-offs would be smaller and, hence, less bandwidth needs to be reserved.

\subsubsection{Comparison among Alternatives without Next-Cell Information}

We now comparatively evaluate the performance of three difference schemes: AC1, AC2, and AC3. Fig. 11 plots $P_{C B}$ and $P_{H D}$. First, in terms of $P_{C B}$, three schemes work almost the same even though AC1 has the smallest $P_{C B}$-with small differences-for the entire offered loads we examined. On the other hand, in terms of $P_{H D}, \mathrm{AC} 2$ and AC3 work almost the same, and AC1 is worse. Our goal is not achieved in a highly overloaded region (say, $L>150$ ) for AC1. $P_{H D}$ does not exceed 0.02 even at the offered load of 300 , which is good because this small violation ratio might be tolerable in most practical applications.

Now, we consider the complexity of these schemes measured in average number of $B_{r}$ calculations for the admission test of a new connection request $\left(=N_{\text {calc }}\right)$. Note that, to calculate $B_{r}$ in a cell, its BS needs to communicate with BSs in all adjacent cells. Fig. 12 shows that $N_{\text {calc }}$ for AC1 is 1, irrespective of the offered load because only the BS of the cell in which the new connection was requested has to calculate $B_{r}$ while $N_{\text {calc }}=3$ for AC2 because BSs in all adjacent cells are required to calculate $B_{r}$. For AC3, which is a hybrid of AC1 and AC2, $N_{\text {calc }}=1$ for low offered load, but it starts to increase at about $L=80$. However, the value is observed to be less than 1.5 in all of our simulations, i.e., less than a half of that of AC2. The complexity increase could be larger for two-dimensional cellular structures. Because AC3 works almost the same as AC2 in terms of $P_{C B}$ while keeping $P_{H D}$ below the target with a lower complexity according to our simulation results, we conclude that $\mathrm{AC} 3$ is a better choice than $\mathrm{AC} 2$.

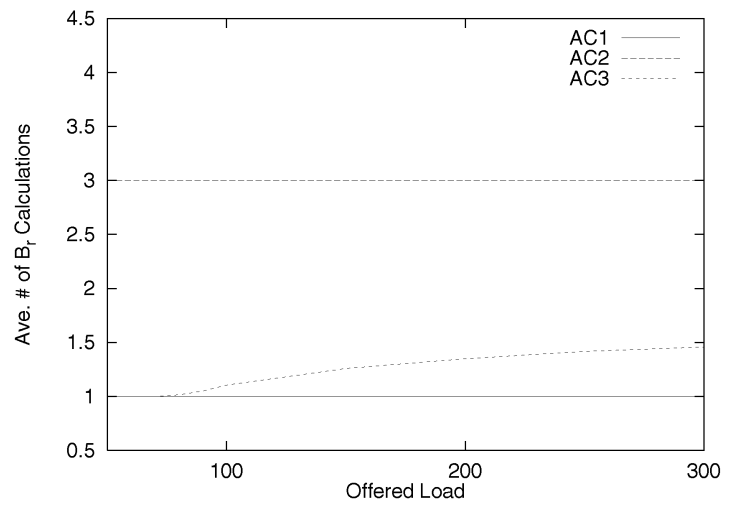

(a)

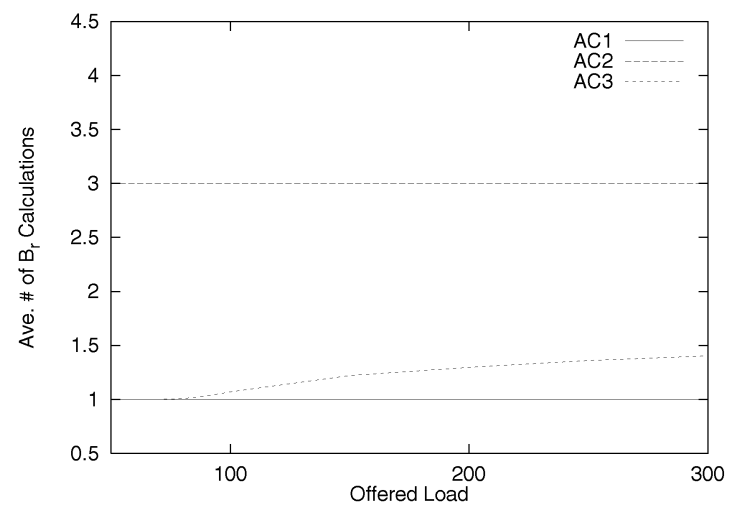

(b)

Fig. 12. Comparison among AC1, AC2, and $\mathbf{A C 3}$ using average number of $B_{r}$ calculations for an admission test versus offered load. (a) High user mobility. (b) Low user mobility. 
TABLE 2

Status in Each Cell at the End of Simulations When the Offered Load is 300 and $R_{v o}=1.0$, and with High User Mobility

\begin{tabular}{|c||c|c|c|c|c|}
\hline Cell & $P_{C B}$ & $P_{H D}$ & $T_{\text {est }}$ & $B_{r}$ & $B_{u}$ \\
\hline 1 & $1.28 \mathrm{e}-1$ & $5.10 \mathrm{e}-3$ & 1 & 1.04 & 89 \\
2 & $9.59 \mathrm{e}-1$ & $1.30 \mathrm{e}-2$ & 45 & 102 & 89 \\
3 & $9.57 \mathrm{e}-1$ & $1.30 \mathrm{e}-2$ & 45 & 93.6 & 97 \\
4 & $2.41 \mathrm{e}-1$ & $6.01 \mathrm{e}-3$ & 1 & 2.13 & 93 \\
5 & $9.79 \mathrm{e}-1$ & $2.58 \mathrm{e}-2$ & 45 & 89.9 & 99 \\
6 & $3.83 \mathrm{e}-1$ & $7.12 \mathrm{e}-3$ & 1 & 1.97 & 87 \\
7 & $9.80 \mathrm{e}-1$ & $2.90 \mathrm{e}-2$ & 45 & 85.8 & 100 \\
8 & $2.75 \mathrm{e}-1$ & $6.97 \mathrm{e}-3$ & 2 & 3.22 & 94 \\
9 & $9.57 \mathrm{e}-1$ & $1.80 \mathrm{e}-2$ & 45 & 10.4 & 88 \\
10 & $9.26 \mathrm{e}-1$ & $1.51 \mathrm{e}-2$ & 45 & 102 & 81 \\
\hline
\end{tabular}

(a)

\begin{tabular}{|c||c|c|c|c|c|}
\hline Cell & $P_{C B}$ & $P_{H D}$ & $T_{e s t}$ & $B_{r}$ & $B_{u}$ \\
\hline 1 & $6.23 \mathrm{e}-1$ & $6.53 \mathrm{e}-3$ & 2 & 5.63 & 97 \\
2 & $6.66 \mathrm{e}-1$ & $6.57 \mathrm{e}-3$ & 2 & 5.44 & 84 \\
3 & $7.30 \mathrm{e}-1$ & $7.54 \mathrm{e}-3$ & 10 & 22.5 & 75 \\
4 & $8.06 \mathrm{e}-1$ & $7.80 \mathrm{e}-3$ & 7 & 19.2 & 73 \\
5 & $7.65 \mathrm{e}-1$ & $7.31 \mathrm{e}-3$ & 10 & 24.2 & 74 \\
6 & $5.65 \mathrm{e}-1$ & $6.74 \mathrm{e}-3$ & 5 & 11.3 & 66 \\
7 & $6.17 \mathrm{e}-1$ & $6.42 \mathrm{e}-3$ & 3 & 5.81 & 89 \\
8 & $8.10 \mathrm{e}-1$ & $6.87 \mathrm{e}-3$ & 3 & 9.18 & 87 \\
9 & $7.68 \mathrm{e}-1$ & $7.42 \mathrm{e}-3$ & 8 & 20.1 & 78 \\
10 & $6.46 \mathrm{e}-1$ & $5.41 \mathrm{e}-3$ & 1 & 4.50 & 90 \\
\hline
\end{tabular}

(b) (a) AC1. (b) AC3.

Now, we compare AC1 with AC3 by examining each cell when the system is overloaded. Table 2 shows the state of each cell at the end of simulations when the offered load is 300 and $R_{v o}=1.0$ for high user mobility with Table 2a AC1 and Table $2 \mathrm{~b}$ AC3. The first column represents the cell number, the second is $P_{C B}$, the third is $P_{H D}$, the fourth is the value of $T_{e s t}$, the fifth is the value of $B_{r}$, and the sixth is the value of $B_{u}$, all at the end of the simulations. FromTable 2b, AC3 is found to work similar throughout all cells in terms of $P_{C B}$ while meeting the constraint $P_{H D} \leq P_{H D \text {,target }}$. $B_{r}$ can change dramatically depending on the traffic condition in adjacent cells even with the same $T_{\text {est }}$ as observed in Table 2 . However, according to Table 2a of AC1, the performance of each cell is found to fluctuate greatly, i.e., the performance in terms of $P_{C B}, P_{H D}, T_{e s t}$, and $B_{r}$ drastically differ in roughly every two cells. This is not fair to those mobiles which want to establish new connections in cells with a very high $P_{C B}$, e.g., cells $\langle 2\rangle,\langle 3\rangle,\langle 5\rangle,\langle 7\rangle,\langle 9\rangle$, and $\langle 10\rangle$ in the table. More importantly, $P_{H D}$ 's of these cells are not bounded. This phenomenon was anticipated as explained in Section 4.4 when the admission test checks the current cell only as was done in AC1.

Table 3 shows the status of each cell at the end of simulations with a different mobility pattern when the offered load $=300$ and $R_{v o}=1.0$. For these simulations, the direction of mobiles are not chosen randomly. Instead, all mobiles follow the direction from cell $<1>$ to cell $<10>$. Moreover, two border cells, i.e., cells $\langle 1\rangle$ and $\langle 10\rangle$, are disconnected. Now, cell $<1>$ won't have any incoming mobiles from adjacent cells. Naturally, $P_{H D}$ will be zero at cell $<1>$. For AC1, we observe a behavior similar to that in Table 3a. Especially, because cell $<1>$ doesn't care about the status of cell $<2>$, the BS of cell $<1>$ accepted all new connection requests, hence $P_{C B}=0$. Cell $<2>$ also doesn't care about the status of cell $<3>$. These make cell $<3>$ overcrowded, and eventually result in a very high $P_{C B}$ (near 1) and overtarget $P_{H D}$ at cell $<3>$. This type of patterns appears every other cell as shown in the table. On the other hand, for AC3, cell $<1>$ cares about cell $<2>$, and blocks some new connection requests. Every cell $<i>$ cares about the status of cell $<i+1>$. Eventually, balanced performance is observed over the entire system while every cell meeting the constraint on $P_{H D}$.

\subsubsection{Adaptive Reservation with Next-Cell Information}

As mentioned earlier, the two-dimensional environment is used to evaluate the adaptive reservation with next-cell information in comparison with the one without such information. Admission control schemes AC3 and AC3 w/ NC are used for the comparison as AC 3 is found to be the best based on the evaluation thus far.

We first illustrate how to predict the next cell from the location information of a mobile using an example. In Fig. 7, a mobile was in cell $C 2$, and eventually moves into cell $C 3$. When the mobile is at location $A$, the next cell can be predicted with probability 1 assuming that the direction of the mobile is also known. Note that the GPS gives both location and direction information. However, the next cell cannot be predicted when the mobile is at location $B$ even if its direction is known since the mobile can change its direction at the intersection. When the direction information is not available, the next cell can be predicted only for some cases even at location $A$, depending on the previous cell of the mobile, i.e., only when the mobile's previous cell is neither $C 3$ nor $C 2$, it can be predicted. Note that this next-cell prediction will depend on the cellular environment such as the road topology and traffic signals/signs in each cell.

In the real world, the next-cell information will be available to only a subset of mobiles. However, we compare three extreme cases to evaluate the advantages of the information: 1) next-cell information is not available for any mobile (referred to as AC3), 2) location/direction information is available for every mobile (referred to as AC3 w/ LOC), and 3 ) path information is available for every mobile (referred to

\section{TABLE 3}

Status in Each Cell at the End of Simulations when the Offered Load is 300, $R_{v o}=1.0$ and All Mobiles Follow One Direction with High Mobility

\begin{tabular}{|c||c|c||c|c|}
\hline \multicolumn{1}{|c||}{} & \multicolumn{2}{c||}{ AC1 } & \multicolumn{2}{c|}{ AC3 } \\
\hline Cell & $P_{C B}$ & $P_{H D}$ & $P_{C B}$ & $P_{H D}$ \\
\hline 1 & 0. & 0. & $5.61 \mathrm{e}-02$ & 0. \\
2 & $5.24 \mathrm{e}-01$ & $6.63 \mathrm{e}-03$ & $5.38 \mathrm{e}-01$ & $4.58 \mathrm{e}-03$ \\
3 & $9.66 \mathrm{e}-01$ & $1.09 \mathrm{e}-02$ & $7.83 \mathrm{e}-01$ & $7.38 \mathrm{e}-03$ \\
4 & $2.84 \mathrm{e}-01$ & $5.98 \mathrm{e}-03$ & $7.06 \mathrm{e}-01$ & $5.78 \mathrm{e}-03$ \\
5 & $9.45 \mathrm{e}-01$ & $4.15 \mathrm{e}-02$ & $6.35 \mathrm{e}-01$ & $5.93 \mathrm{e}-03$ \\
6 & $4.17 \mathrm{e}-01$ & $7.28 \mathrm{e}-03$ & $7.44 \mathrm{e}-01$ & $7.96 \mathrm{e}-03$ \\
\hline
\end{tabular}




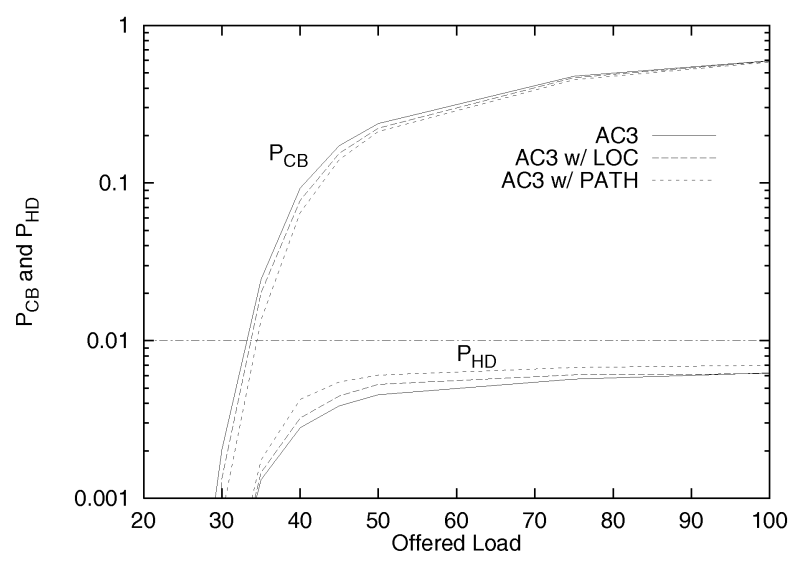

(a)

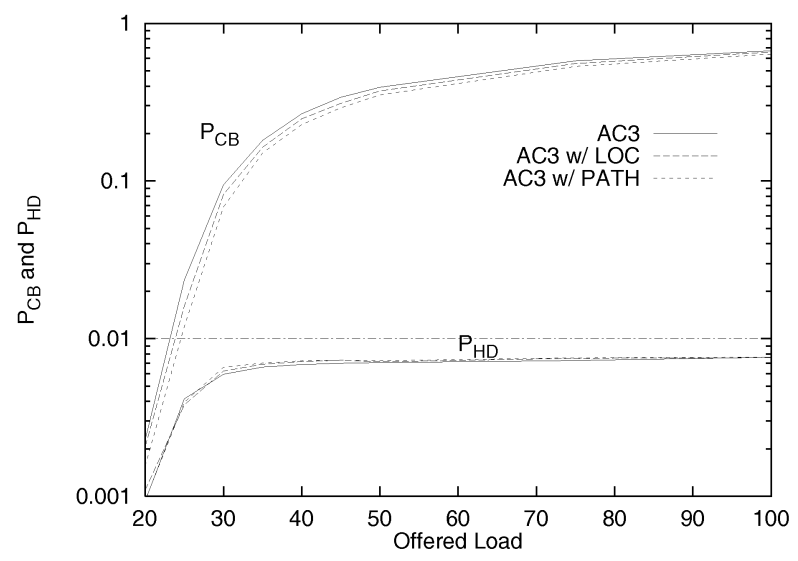

(b)

Fig. 13. Comparison of three cases: $P_{C B}$ and $P_{H D}$ versus offered load. (a) $R_{v 0}=1.0$. (b) $R_{v 0}=5.0$.

as AC3 w/ PATH). Note that admission control AC3 w/ NC can be always (sometimes) used in case of AC3 w/ $\operatorname{PATH}(\mathrm{AC} 3$ w/ LOC).

Fig. 13 plots $P_{C B}$ and $P_{H D}$ of three cases as the offered load increases. First, $P_{H D}$ is bounded for all three cases, thus achieving the design goal. As expected, the performance in terms of $P_{C B}$ is shown in the order of AC3 w/ PATH, AC3 w/ LOC, and AC3, i.e., more new connections can be admitted in that order. Even though the differences are not significant, we can determine that location/path information is quite advantageous.

Now, let us consider the computation complexity of the admission control schemes by comparing the numbers of numerical operations (including summations and multiplications) and comparisons for an admission decision. Comparisons include decisions such as if $t_{s o j}$ is larger than a value in summations of (4) and (8). We did not include the complexity and cost for the interface between the navigation systems and the network. Fig. 14 shows the average numbers of numerical operations and comparisons for an admission decision for three cases. We observe the complexity gap between AC3 w/ PATH and the other two is significant while AC3 w/ LOC is about 20 percent (30 percent) better than AC3 in terms of the number of operations (comparisons).

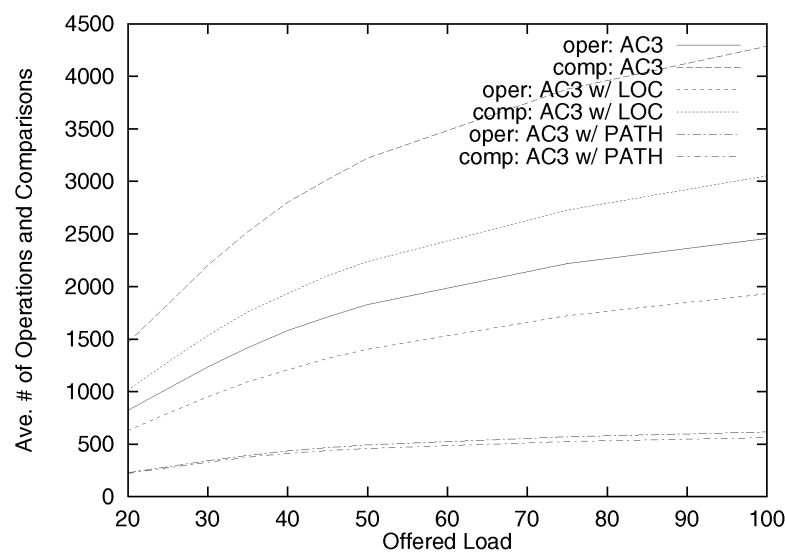

(a)
The scheme without next-cell information may look too complicated to be useful. Note, however, that the operations and comparisons are distributed over a number of cells since five cells participate in the $B_{r}$ calculation in a cell and more than one $B_{r}$ are calculated for an admission decision. This complexity number will also drop if a smaller $N_{\text {quad }}$ is used. Interestingly, the complexity for $R_{v o}=0.5$ is much smaller than that for $R_{v o}=1.0$. This is because the admission decision complexity depends on the number of existing connections in the current and adjacent cells, but for $R_{v o}=0.5$, there are fewer existing connections in the system on average for a given offered load.

\subsection{Time-Varying Traffic/Mobility}

Now, we vary the connection generation rate $\lambda$ and speed range $\left[S P_{\min }, S P_{\max }\right]$ over time. Each simulation is run for two days in simulation time. Fig. 15a shows time-varying averages of mobiles' speeds and offered loads. First, for a given value of the average speed (marked by $S$ ), the speed range is given by $[S-20, S+20](\mathrm{km} / \mathrm{h})$. Second, the original offered load (marked by $L_{o}$ ) is the traffic load from the new connections generated, which is the offered load $L$ defined in (9). In this time-varying case, a blocked connection request will be rerequested with probability $1-0.1 N_{\text {ret }}$ after waiting

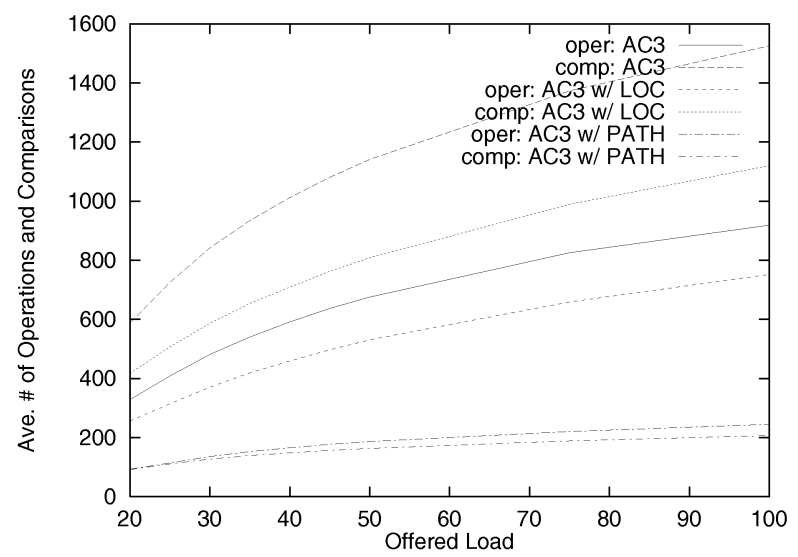

(b)

Fig. 14. Comparison of three cases: average number of numerical operations and comparisons versus offered load. (a) $R_{v 0}=1.0$. (b) $R_{v 0}=5.0$. 


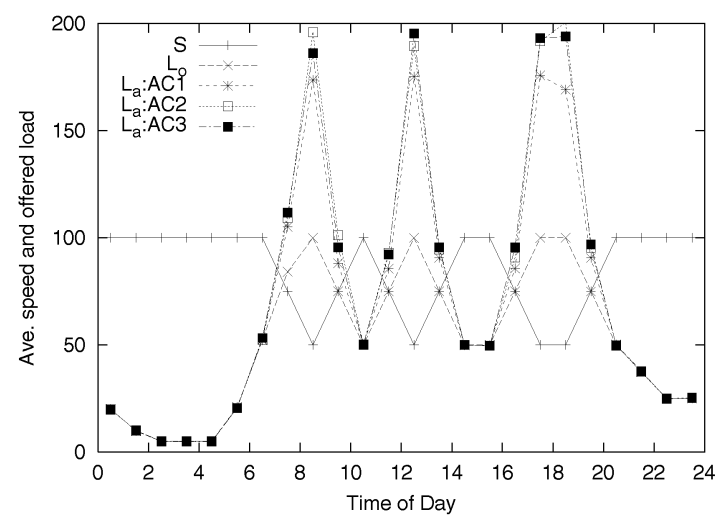

(a)

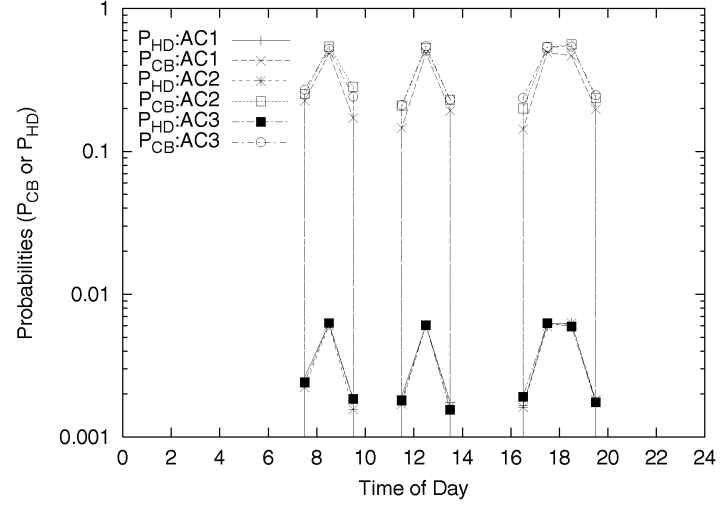

(b)

Fig. 15. Simulation results for the time-varying case: (a) speed and offered load, mobiles' average speed and offered load versus time of day and (b) probabilities, $P_{C B}$ and $P_{H D}$ versus time of day.

five seconds, where $N_{\text {ret }}$ is the number of times a connection request has been made. So, depending on $P_{C B}$, the actual offered load $L_{a}$ will vary, i.e., the larger $P_{C B}$, the larger $L_{a}$. From the figure, we observe that the values of $L_{a}$ for different schemes are different when the system is highly-loaded even with the same $L_{o}$. Note that the fluctuations of the offered load and speed represent the reality, that is, the offered load peaks during rush hours (e.g., around 9 a.m., 1 p.m., and 5-6 p.m.) at low speeds. Fig. $15 \mathrm{~b}$ shows $P_{C B}$ and $P_{H D}$ over time of day for three different schemes. We don't consider the schemes with next-cell information here. The probability samples represent the average probability during the corresponding one-hour period, i.e., $P_{C B}$ at $t=8.5$ represents the average over the time interval $[8,9]$. First, we observe that outside the peak hour regions, both $P_{C B}$ and $P_{H D}$ are negligibly small. During the peak hours, $P_{H D}$ is almost the same for different schemes, and bounded by $P_{H D \text {,target }}(=0.01)$. On the other hand, $P_{C B}$ of AC1 is found to be lower than that of the other two schemes, and the differences between $P_{C B}$ 's of AC1 and AC3 are larger compared to those from the stationary case in Fig. 11. This is due to the positive feedback effect of the offered load increase; that is, from the original offered load, the difference between AC1 and AC 3 could be small, but this small difference could be amplified through the retrials of each blocked connection request.

According to the results of the time-varying case, AC1 is the best because it yields the lowest $P_{C B}$ while meeting our goal. For this time-varying case, we considered only a regular traffic pattern: a high offered load for relatively short peak-hour periods (of one or two hours). However, AC1 may have undesirable behaviors as previously observed in the time-invariant case, because there might be unexpected irregular traffic and mobility patterns in the real world. AC3 was found to be robust in many different scenarios with relatively low complexity (up to 1.5 times that of AC1 in our simulations). So, AC3 and AC3 w/ NC are the most favorable among the schemes considered.

\section{Related Work}

The notion of reserving channels for hand-offs was introduced in the mid-1980s [7]. In this scheme, a set of channels are permanently reserved in advance for hand-offs. It was shown that this static reservation is optimal in the sense of minimizing a linear objective function of the connection blocking probability and the hand-off dropping probability when both new and hand-off connection arrivals are Poisson, and connection durations are exponentially-distributed [18].

Most existing bandwidth-reservation schemes for handoffs assume that the hand-off connection arrivals are Poisson, and each connection requires an identical amount of bandwidth with an exponentially-distributed sojourn time in each cell. It is known that the sojourn time of handed-off connections is not really exponentially-distributed [8], [6]. In the method of [13], each base station measures the average rate of actual hand-off connection arrivals and then uses an $\mathrm{M} / \mathrm{M} / 1$ queueing model to estimate the number of radio channels required for handoffs. The number of required radio channels is modelled as the number of buffers in the $\mathrm{M} / \mathrm{M} / 1$ queue. Consequently, this method can only handle connections with identical bandwidth demands. Other methods that use estimated average hand-off and new connection arrival rates to estimate the number of radio channels to be reserved for hand-offs can be found in [15], [9].

We are not the first to attempt to design bandwidthreservation and admission-control schemes to keep the connection hand-off dropping probability below a target value. The authors of [14] advocated the connection handoff dropping probability as an important connection-level QoS parameter in wireless/mobile networks, and designed a distributed call admission-control scheme to keep the connection hand-off dropping probability below a specified limit. With their scheme, the BS obtains the required bandwidth for both the existing and hand-off connections after a certain time interval, then performs admission control so that the required bandwidth may not exceed the cell capacity. Their scheme was shown to be better than the static reservation scheme. The authors of [12] extended this scheme as a part of their proposal to accommodate heterogeneous connection bandwidths and studied the effects of design parameters used in the scheme. The main problems of these schemes are: 1) they assumed the sojourn time of each mobile is exponentially-distributed, which is impractical. Moreover, it is not clear whether the scheme will still work when this assumption does not hold and 
2) there is no specified mechanism to predict which cells mobiles will move to.

The shadow cluster concept was suggested in [11] to estimate future resource requirements and perform admission control in order to limit the hand-off dropping probability, in which the shadow cluster is a set of cells around an active mobile. This scheme is based on the precise knowledge of each user mobility, depending on the location and time, which they assumed given. Our mobility estimation can provide the knowledge of mobility used in their scheme, but it is unclear how it will work if the knowledge is not accurate. (This may be the case if our cellspecific history-based mobility estimation is used.) How to determine the shadow cluster is also not defined clearly. Moreover, their scheme is computationally too expensive to be practical.

Our scheme is more realistic than the above-mentioned schemes, because 1) exponentially-distributed mobile sojourn times are not assumed, instead, mobiles' hand-off behaviors are estimated based on a history of observations in each cell, 2) our scheme is robust to the inaccuracy of mobility estimation and the time-variation of traffic/ mobility, thanks to our mobility estimation time window control, and 3) due to the adaptability of our scheme, it is not required to determine the optimal value of parameters, which might depend on the traffic status, as in [12].

There were also limited efforts to estimate mobility. The authors of [12] explored mobility estimation for an indoor wireless system based on both mobile-specific and cellspecific observation histories. Mobile-specific observation of mobility is costly and not accurate in general. Our mobility estimation not only predicts the next cell to which a mobile will move, but also estimates the hand-off time (or sojourn time). This hand-off time estimation makes it possible for BSs to reserve bandwidth more efficiently.

There have also been research efforts for adaptive bandwidth reservation. The author of [10] suggested bandwidth reservation depending on the existing connections in adjacent cells. However, the scheme lacks such details as how much of bandwidth should be reserved. The bandwidth-reservation and admission-control schemes in [19] assume that the mobility of users is predictable, that is, mobility can be characterized by the set of cells the mobile is expected to visit during the lifetime of the mobile's connection. This assumption is more or less similar to the next-cell information in our scheme, and does not hold for most wireless/mobile networks. Moreover, the scheme reserves the required bandwidth at every cell and node in the mobility specification, which is usually excessive.

\section{CONCLUSION}

In this paper, we designed and evaluated predictive, adaptive bandwidth reservation for hand-offs and admission control so as to keep the hand-off dropping probability below a prespecified value. Our schemes utilize the following two components to reserve bandwidth for hand-offs:

1. hand-off estimation functions which are used to predict a mobile's next cell and estimate its sojourn time probabilistically based on its previously-resided cell and the observed history of hand-offs in each cell and

2. mobility estimation time window control scheme in which, depending on the observed hand-off drops, the estimation time window size is controlled adaptively for efficient use of bandwidth and effective response to

a. time-varying traffic/mobility and

b. inaccuracy of mobility estimation.

We considered three different admission-control schemes depending on how many neighboring BSs participate in the admission decision of a new connection request. Through the performance and complexity comparisons, we concluded a hybrid one is superior to the others. Our best scheme is not optimal in the sense that there might be a better scheme resulting in a lower connection blocking probability while keeping the hand-off dropping probability below the target value. However, this scheme is not complex nor based on any impractical assumptions and, hence, it is readily implementable. It is also shown to be robust and work well under a variety of traffic loads, connection bandwidths, and mobility.

We also explored how to utilize path/location information readily available from ITS navigation systems or GPS for our bandwidth reservation and admission control. Path/location information is found to be useful in the sense of 1) admitting more new connections by reserving bandwidth for hand-offs more efficientl and 2) requiring less computational complexity for admission decisions.

\section{ACKNOWLEDGMENTS}

A subset of materials in this paper was presented at Association for Computing Machinery's Special Interest Group on Data Communication (SIGCOMM), Vancouver, British Columbia, September 1998 and IEEE Wireless Communications and Network Conference (WCNC '00), Chicago, Illinois, September 2000. The work reported in this paper was supported in part by the US Air Force Office of Scientific Research under Grant No. F49620-00-1-0327 and the US Office of Naval Research under Grant No. N0001499-1-0465. Any opinions, findings, and conclusions or recommendations expressed in this publication are those of the authors and do not necessarily reflect the views of the funding agencies.

\section{REFERENCES}

[1] H. Ahmadi, A. Krishna, and R.O. LaMaire, "Design Issues in Wireless LANs," J. High-Speed Networks, vol. 5, no. 1, pp. 87-104, 1996.

[2] S. Choi and K.G. Shin, "A Cellular Local Area Network with QoS Guarantees for Heterogeneous Traffic," Proc. IEEE INFOCOM '97, pp. 1032-1039, Apr. 1997.

[3] S. Choi and K.G. Shin, "Uplink CDMA Systems with Diverse QoS Guarantees for Heterogeneous Traffic," Proc. ACM/IEEE MobiCom '97, pp. 120-130, Sept. 1997.

[4] D. Collins and C. Smith, 3G Wireless Networks. McGraw-Hill, 2001

[5] Enhanced 911, FCC website at http://www.fcc.gov/e911. 2002.

[6] Y. Fang and I. Chlamtac, "A New Mobility Model and Its Application in the Channel Holding Time Characterization in PCS Networks," Proc. IEEE INFOCOM '99, Mar. 1999. 
[7] D. Hong and S.S. Rappaport, "Traffic Model and Performance Analysis for Cellular Mobile Radio Telephone Systems with Prioritized and Nonprioritized Procedures," IEEE Trans. Vehicular Technology, vol. 35, no. 3, pp. 77-92, Aug. 1986.

[8] C. Jedrzycki and V.C.M. Leung, "Probability Distributions of Channel Holding Time in Cellular Telephony Systems," Proc. IEEE Vehicular Technology Conf. (VTC '96), May 1996.

[9] S. Kim and T.F. Znati, "Adaptive Handoff Channel Management Schemes for Cellular Mobile Communication Systems," Proc. IEEE Int'l Conf. Comm. (ICC '99), 1999.

[10] K. Lee, "Supporting Mobile Multimedia in Integrated Service Networks," ACM Wireless Networks, vol. 2, pp. 205-217, 1996.

[11] D.A. Levine, I.F. Akyildiz, and M. Naghshineh, "A Resource Estimation and Call Admission Algorithm for Wireless Multimedia Networks Using the Shadow Cluster Concept," IEEE/ACM Trans. Networking, vol. 5, no. 1, pp. 1-12, Feb. 1997.

[12] S. Lu and V. Bharghavan, "Adaptive Resource Management Algorithms for Indoor Mobile Computing Environments," Proc. ACM SIGCOMM '96, pp. 231-242, Aug. 1996.

[13] X. Luo, I. Thng, and W. Zhuang, "A Dynamic Pre-Reservation Scheme for Handoffs with GoS Guarantee in Mobile Networks," Proc. IEEE Int'l Symp. Computers and Comm., July 1999.

[14] M. Naghshineh and M. Schwartz, "Distributed Call Admission Control in Mobile/Wireless Networks," IEEE J. Selected Areas in Comm., vol. 14, no. 4, pp. 711-717, May 1996.

[15] L. Ortigoza-Guerrero and A. H. Aghvami, "A Prioritized Handoff Dynamic Channel Allocation Strategy for PCS," IEEE Trans. Vehicular Technology, vol. 48, no. 4, July 1999.

[16] A. Papoulis, Probability, Random Variables, and Stochastic Processes, third ed. McGraw-Hill, 1991.

[17] A.K. Parekh and R.G. Gallager, "A Generalized Processor Sharing Approach to Flow Control in Integrated Services Networks: The Single-Node Case," IEEE/ACM Trans. Networking, vol. 1, no. 3, pp. 344-357, June 1993.

[18] R. Ramjee, R. Nagarajan, and D. Towsley, "On Optimal Call Admission Control in Cellular Networks," Proc. IEEE INFOCOM '96, pp. 43-50, 1996.

[19] A.K. Talukdar, B.R. Badrinath, and A. Acharya, "On Accommodating Mobile Hosts in an Integrated Services Packet Network," Proc. IEEE INFOCOM '97, pp. 1048-1055, Apr. 1997.

[20] A.J. Viterbi, CDMA: Principles of Spread Spectrum Communication. Reading, Mass.: Addison-Wesley, 1995.

[21] Y. Zhao, Vehicle Location and Navigation Systems. Artech House, 1997.

[22] Q. Zheng and K. G. Shin, "On the Ability of Establishing RealTime Channels in Point-to-Point Packet-Switched Networks," IEEE Trans. Comm., vol. 42, nos. 2/3/4 pp. 1096-1105, Feb./Apr., 1994.

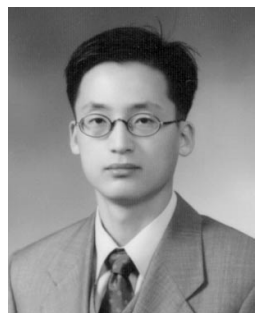

Sunghyun Choi (S'96-M'00) received the BS (summa cum laude) and MS degrees in electrical engineering from Korea Advanced Institute of Science and Technology (KAIST) in 1992 and 1994, respectively, and received PhD from the Department of Electrical Engineering and Computer Science in the University of Michigan, Ann Arbor in September, 1999. He is a senior member Research Staff at Philips Research, Briarcliff Manor, New York. His current research interests are in the area of wireless/mobile networks with emphasis on the QoS guarantee and adaptation, in-home multimedia networks, wireless LAN and PAN, MAC protocols, connection and mobility management, and multimedia CDMA. He has authored/coauthored more than 25 technical papers and book chapters in the areas of wireless/mobile networks and communications. Currently, he is also an active participant and contributor of IEEE 802.11 Working Group standardization. Dr. Choi was a recipient of the Korea Foundation for Advanced Studies Scholarship and the Korean Government Overseas Scholarship during 1997-1999 and 1994-1997, respectively. He is also a winner of the Humantech Thesis Prize from Samsung Electronics in 1997. He is a member of the IEEE.

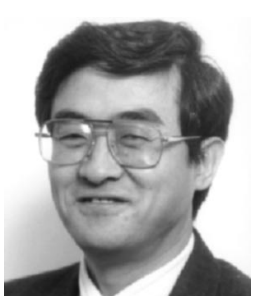

Kang G. Shin (S'75-M'78-SM'83-F'92) received the BS degree in electronics engineering from Seoul National University, Seoul, Korea in 1970, and the MS and PhD degrees in electrical engineering from Cornell University, Ithaca, New York in 1976 and 1978, respectively. He is the Kevin and Nancy O'Connor Professor of Computer Science and Founding Director of the Real-Time Computing Laboratory in the Department of Electrical Engineering and Computer Science,University of Michigan, Ann Arbor, Michigan. His current research focuses on QoS-sensitive networking and computing as well as on embedded real-time OS, middleware and applications, all with emphasis on timeliness and dependability. He has supervised the completion of $42 \mathrm{PhD}$ theses, and authored/coauthored more than 500 technical papers and numerous book chapters in the areas of distributed real-time computing and control, computer networking, fault-tolerant computing, and intelligent manufacturing. He has coauthored (jointly with C.M. Krishna) a textbook "Real-Time Systems," McGraw Hill, 1997. $\mathrm{He}$ received the Outstanding IEEE Transactions on Automatic Control Paper Award in 1987, Research Excellence Award in 1989, Outstanding Achievement Award in 1999, Service Excellence Award in 2000, and Distinguished Faculty Achievement Award in 2001 from The University of Michigan. He also coauthored papers with his students which received the Best Student Paper Awards from the 1996 IEEE RealTime Technology and Application Symposium, and the 2000 UNSENIX Technical Conference. From 1978 to 1982, he was on the faculty of Rensselaer Polytechnic Institute, Troy, New York. He has held visiting positions at the US Airforce Flight Dynamics Laboratory, AT\&T Bell Laboratories, Computer Science Division within the Department of Electrical Engineering and Computer Science at the University of California at Berkeley, and the International Computer Science Institute, Berkeley, California, the IBM T.J. Watson Research Center, and the Software Engineering Institute at Carnegie Mellon University. He has also chaired the Computer Science and Engineering Division, EECS Department, The University of Michigan for three years beginning January 1991. He is fellow of IEEE, a member of the IEEE Computer Society and ACM, and a member of the Korean Academy of Engineering. He was the general chair of the 2000 IEEE Real-Time Technology and Applications Symposium, the program chair of the 1986 IEEE Real-Time Systems Symposium (RTSS), the general chair of the 1987 RTSS, the guest editor of the 1987 August special issue of IEEE Transactions on Computers on Real-Time Systems, a program cochair for the 1992 International Conference on Parallel Processing, and served numerous technical program committees. He also chaired the IEEE Technical Committee on Real-Time Systems during 1991-93, was a Distinguished Visitor of the Computer Society of the IEEE, an editor of IEEE Transactions on Parallel and Distributed Computing, and an Area Editor of International Journal of Time-Critical Computing Systems, Computer Networks, and ACM Transactions on Embedded Systems. 